\title{
The Algebraic K-theory of Extensions of a Ring by Direct Sums of Itself
}

\author{
Ayelet Lindenstrauss \\ Department of Mathematics \\ Indiana University \\ Bloomington IN 47405 \\ ayelet@math.indiana.edu \\ Randy McCarthy* \\ Department of Mathematics \\ University of Illinois at Urbana-Champaign \\ Urbana IL 61801 \\ randy@math.uiuc.edu
}

\begin{abstract}
We calculate $K\left(A \ltimes\left(A^{\oplus k}\right)\right)_{p}^{\wedge}$ when $A$ is a perfect field of characteristic $p>0$, generalizing the $k=1$ case $K(A[\epsilon])_{p}^{\wedge}$ which was calculated by Hesselholt and Madsen by a different method in [6]. We use $W(A ; M)$, a construction which can be thought of as topological Witt vectors with coefficients in a bimodule. For a ring or more generally an FSP $A, W\left(A ; M \otimes S^{1}\right) \simeq \tilde{K}(A \ltimes M)$. We give a sum formula for $W\left(A ; M_{1} \oplus \cdots \oplus M_{n}\right)$, and a splitting of $W(A ; M)_{p}^{\wedge}$ analogous to the splitting of the algebraic Witt vectors into a product of p-typical Witt vectors after completion at $p$. We construct an $E^{1}$ spectral sequence converging to $\pi_{*} W^{(p)}(A ; M \otimes X)$, where $W^{(p)}$ is the topological version of $p$ typical Witt vectors with coefficients. This enables us to complete the calculation of $K\left(A \ltimes\left(A^{\oplus k}\right)\right)_{p}^{\wedge}$ in terms of $W^{(p)}(A ; A)$ if the homotopy of the latter is concentrated in dimension 0; for perfect fields of characteristic $p>0$, Hesselholt and Madsen showed in [6] that this condition holds. Using our methods we also give a complete calculation of $W(A ; M)$ where $A$ is a commutative ring and $M$ a symmetric, flat $A$-bimodule whose homotopy groups are vector spaces over $\mathbb{Q}$, and a way of calculating $\tilde{K}(\mathbb{Z} \ltimes \mathbb{Q})$ different than Goodwillie's original one in [7].
\end{abstract}

\section{$\S 0$. Introduction}

For a ring $A$ and an $A$-bimodule $M$, we can look at the trivial square zero extension $A \ltimes M$, where elements of $A$ multiply elements of $M$ using the bimodule structure, and $M \cdot M=0$. Since the ring $A \ltimes M$ contains the ring $A$ as a retract, $K(A)$ is a retract of $K(A \ltimes M)$, and we can write

$$
K(A \ltimes M) \simeq K(A) \times \tilde{K}(A \ltimes M) .
$$

In the course of proving that stable algebraic K-theory agrees with THH in [2], Dundas and McCarthy introduced algebraic K-theory with coefficients in a bimodule, $K(A ; M)$. They describe an equivalence $K(A \ltimes M) \simeq K(A ; B . M)$. This equivalence is functorial in

\footnotetext{
* Partially supported by NSF grant DMS 03-06429
} 
$M$, so splitting $K(A)=K(A ; 0)$ (which is known for $A$ a perfect field of characteristic $p$ ) off from both sides we get

$$
\tilde{K}(A \ltimes M) \simeq \tilde{K}(A ; B . M) \simeq \tilde{K}\left(A ; M \otimes S^{1}\right)
$$

(see the beginning of Section 4 below for the definition of $M \otimes X$ ). In the paper [8], we show that for a connected finite simplicial set $X, \tilde{K}(A ; M \otimes X)$ is equivalent to a 'topological Witt vectors with coefficients' construction we call $W(A ; M \otimes X)$.

'Topological Witt vectors' were originally introduced in [1]. For a ring or more generally an FSP $A$, the topological Hochschild homology $\mathrm{THH}(A)$ has a Connes cyclic structure, and one can look at the homotopy inverse limit of the fixedpoints $\operatorname{THH}(A)^{C_{n}}$ over all positive integers with respect to what are called restriction maps $\operatorname{THH}(A)^{C_{t n}} \rightarrow \operatorname{THH}(A)^{C_{n}}$. (This was called $\operatorname{TR}(A)$ in [1] and [6]; topological cyclic homology involves looking at the inclusion maps $\mathrm{THH}(A)^{C_{t n}} \rightarrow \mathrm{THH}(A)^{C_{n}}$ as well.) Introducing coefficients in a bimodule spoils the Connes cyclic structure, but in [8] we defined simplicial spectra with

$$
U^{n}(A ; M)_{j}=\underbrace{M \wedge \underline{A}^{\wedge j} \wedge \cdots \wedge \underline{M} \wedge \underline{A}^{\wedge j}}_{n \text { times }} .
$$

For each $n, U^{n}(A ; M)$ has a $C_{n}$ action, and there are restriction maps $U^{t n}(A ; M)^{C_{t n}} \rightarrow$ $U^{n}(A ; M)^{C_{n}}$ which agree with the original restriction maps when $A=M$.

The spectrum $W(A ; M)$ is then the homotopy inverse limit of all the $U^{n}(A ; M)^{C_{n}}$ for positive integers $n$ with respect to the restriction maps. We also look at $W_{n_{0}}(A ; M)$, the homotopy inverse limit over the full subcategory on $\left\{1,2, \ldots, n_{0}\right\}$. The homotopy fiber of the map $W_{n_{0}}(A ; M) \rightarrow W_{n_{0}-1}(A ; M)$ induced by restricting the subcategory is $U^{n_{0}}(A ; M)_{h C_{n_{0}}}$.

If we do this for the bimodule $M \otimes X$ in place of $M$, where $X$. is a simplicial set, we get that the $n$ 'th layer in the tower consisting of the $W_{n}(A ; M \otimes X)$ (with the maps induced by category restriction) which is $U^{n}(A ; M \otimes X)_{h C_{n}}$ is an $n$ 'th degree homogenous functor in the sense of Goodwillie's calculus of functors. In [8] we show that this tower is the Taylor tower of the functor $X \rightarrow \tilde{K}(A ; M \otimes X)$, which coverges for obvious degree reasons when $X$ is connected.

In this paper we show that the tower splits when all the $U^{n}(A ; M)$ are rational (which in practice means that the $\pi_{*}(M)$ are rational vector spaces). Otherwise, after completion at $p$ a partial splitting occurs: there is no interaction between layer $n_{1}$ and layer $n_{2}$ unless $n_{1}$ and $n_{2}$ have the same prime-to- $p$ part. This is analogous to the splitting of the algebraic Witt vectors into a product of copies of the $p$-typical Witt vectors, and was done by Hesselholt and Madsen in the $A=M$ case in [6]. We show that for any FSP $A$ and $A$-bimodule $M$,

$$
W(A ; M)_{p}^{\wedge} \simeq \prod_{(t, p)=1}\left(\left(\operatorname{holim}_{\infty \leftarrow n} U^{t p^{n}}(A ; M)^{C_{p^{n}}}\right)_{p}^{\wedge}\right)_{h C_{t}}
$$

where $\operatorname{holim}_{\infty \leftarrow n} U^{t p^{n}}(A ; M)^{C_{p^{n}}} \simeq W^{(p)}\left(A ; M^{\hat{\otimes}_{A} t}\right)$ nonequivariantly. Here $W^{(p)}$ plays the role of topological $p$-typical Witt vectors with coefficients; as in the algebraic case, it is 
easier to calculate. See equation (1.0.5) below for the definition of the derived tensor product of modules over a functor with smash product.

In two applications where there is splitting, namely when $A$ is a commutative ring and $M$ a symmetric, flat $A$-bimodule whose homotopy groups are vector spaces over $\mathbb{Q}$ (Claim 3.3 below) and for $\tilde{K}(\mathbb{Z} \ltimes \mathbb{Q}) \simeq W\left(\mathbb{Z} ; \mathbb{Q} \otimes S^{1}\right.$ ) (calculated originally by Goodwillie and discussed here at the end of the paper), we complete the calculation of $W$.

But for the main focus of our interest, $\tilde{K}(A \ltimes M) \simeq W\left(A ; M \otimes S^{1}\right)$ for $M$ not necessarily rational, the splitting (0.0.2) still leaves the problem of calculating $W^{(p)}(A ;(M \otimes$ $\left.\left.S^{1}\right)^{\hat{\otimes}_{A} t}\right) \simeq \operatorname{holim}_{\infty \leftarrow n} U^{t p^{n}}\left(A ; M \otimes S^{1}\right)^{C_{p^{n}}}$ and the effect of taking the $C_{t}$ homotopy quotient on it. In the case $M=A^{\oplus k}$, however, we use a sum formula we give in Section 2 for $W\left(A ; M_{1} \oplus \cdots \oplus M_{k}\right)$ to decompose $W\left(A ; A^{\oplus k} \otimes S^{1}\right)$ into a product of $W\left(A ; A \otimes S^{a}\right)$ 's. These break down by $(0.0 .2)$. In Section 4 we develop a spectral sequence (Corollary 4.11)

$$
E_{r, *}^{1}=\pi_{*-r}\left(\operatorname{holim}_{\infty} U^{p^{n+r}}(A ; M)^{C_{p^{n}}} \wedge_{C_{p^{r}}} X^{\wedge p^{r}} / X^{\wedge p^{r-1}}\right) \Rightarrow \pi_{*} W^{(p)}(A ; M \otimes X),
$$

where $C_{p^{r}}$ acts on $X^{\wedge p^{r}}$ by permuting coordinates and $X^{\wedge p^{r-1}}$ sits inside $X^{\wedge p^{r}}$ as the $C_{p}$ fixedpoints. We use this spectral sequence in Section 5 to compute $\pi_{*}\left(W^{(p)}\left(A ; M \otimes S^{a}\right)\right)$ in terms of $\pi_{*}\left(W^{(p)}\left(A ; M^{\hat{\otimes}_{A} p^{r}}\right)\right)$ for $r \geq 0$ if all the $W^{(p)}\left(A ; M^{\hat{\otimes}_{A} p^{r}}\right)$ have their homotopy concentrated in dimension 0 . We know this to hold when $M=A$ is a field perfect field of characteristic $p$ by [6], and for a finite sum of these by our sum formula (Corollary 2.3 below). In the $M=A$ case, $\pi_{0}\left(W^{(p)}(A ; A)\right)$ is the ring of $p$-typical Witt vectors of $A$.

Section 6 analyzes the effect of taking homotopy quotients in (0.0.2), and Section 7 assembles the results of the preceding ones. By Theorem (7.2), for any FSP $A$ and prime $p$,

$$
\tilde{K}\left(A \ltimes\left(A^{\oplus k}\right)\right)_{p}^{\wedge} \simeq W\left(A ; A^{\oplus k} \otimes S^{1}\right)_{p}^{\wedge} \simeq \prod_{i=1}^{\infty}\left(W^{(p)}\left(A ; A \otimes S^{i}\right)_{p}^{\wedge}\right)^{m_{i}}
$$

where for $i$ odd or if $p=2$,

$$
m_{i}=\sum_{\substack{t \mid i \\(t, p)=1}} \frac{t}{i} \sum_{\left.d\right|^{\frac{i}{t}}} \mu\left(\frac{i}{t d}\right) k^{d}
$$

while for $i$ even and $p$ odd,

$$
m_{i}=\sum_{\substack{t \mid \frac{i}{2} \\(t, p)=1}} \frac{t}{i} \sum_{d \mid \frac{i}{t}} \mu\left(\frac{i}{t d}\right) k^{d}
$$

for the Möbius $\mu$-function (see (7.1.1)). The homotopy groups of each $W^{(p)}\left(A ; A \otimes S^{i}\right)$ are given in Corollary (5.4), and when $A$ is the FSP associated to a perfect field of characteristic $p>0$, they take the particularly simple form

$$
\pi_{*}\left(W^{(p)}\left(A ; A \otimes S^{a}\right)\right) \cong \begin{cases}0 & \text { if } *<a \text { or } *-a p \text { odd } \\ W(A) / p^{r} W(A) & \text { if } a p^{r-1}<* \leq a p^{r}, *-a p \text { even }\end{cases}
$$


if $p$ is odd or $a$ is even, and

$$
\pi_{*}\left(W^{(2)}\left(A ; A \otimes S^{a}\right)\right) \cong \begin{cases}0 & \text { if } *<a \text { or } * \text { is even } \\ W(A) / 2 W(A) & \text { if } * \geq a \text { is odd }\end{cases}
$$

when $p=2$ and $a$ is odd by Corollaries (5.5) and (5.6) below.

We thank Morten Brun for his comments on an earlier draft of this paper, and the referee for his careful reading of the manuscript and his many useful suggestions.

\section{$\S 1$. Preliminaries}

We recall the definitions from [8] which will be used here; details can be found in [8], where the constructions are done more generally for functors over a category. We will not be working over a category in this paper, although the main technical results like Theorems (2.2) and (3.3) and Corollary (5.3) can be stated and proved over a category with the obvious adjustments to the given proofs.

A functor with stabilization is a functor $F$ from the category $\mathcal{S}_{*}$ of pointed simplicial sets to itself, sending $n$-connected spaces to $n$-connected spaces, along with a natural transformation $\lambda_{X, Y}: X \wedge F(Y) \rightarrow F(X \wedge Y)$ for all $X, Y \in \mathcal{S}_{*}$ such that

$$
\lambda_{S^{0}, X}: S^{0} \wedge F(X) \rightarrow F\left(S^{0} \wedge X\right)
$$

is the obvious isomorphism for all $X \in \mathcal{S}_{*}$, and such that for all $X, Y, Z \in \mathcal{S}_{*}$,

$$
\lambda_{X, Y \wedge Z} \circ\left(\operatorname{id}_{X} \wedge \lambda_{Y, Z}\right)=\lambda_{X \wedge Y, Z}
$$

We also require of a functor with stabilization $F$ that for every $X$ and $n$, the limit system

$$
\pi_{n}|F(X)| \stackrel{\sigma_{X}}{\longrightarrow} \pi_{n} \Omega|F(\Sigma X)| \stackrel{\sigma_{\Sigma X}}{\longrightarrow} \pi_{n} \Omega^{2}\left|F\left(\Sigma^{2} X\right)\right| \longrightarrow \cdots
$$

should stabilize, where $\sigma_{X}:|F(X)| \rightarrow \Omega|F(\Sigma X)|$ is the adjoint to the map induced on the realizations by $\lambda_{S^{1}, X}$.

A functor with smash product (FSP) is a functor with stabilization $F$ with natural unit and multiplication transformations

$$
\begin{aligned}
& \mathbf{1}_{X}: X \rightarrow F(X) \\
& \mu_{X, Y}: F(X) \wedge F(Y) \rightarrow F(X \wedge Y)
\end{aligned}
$$

for all $X, Y \in \mathcal{S}_{*}$ where the multiplication is associative, and the stabilization $\lambda_{X, Y}$ is given by multiplication with $\mathbf{1}_{X}$. Note that it follows from these conditions that the product of an FSP satisfies $\mu_{X, Y}\left(\mathbf{1}_{X}, \mathbf{1}_{Y}\right)=\mathbf{1}_{X \wedge Y}$ for all $X, Y \in \mathcal{S}_{*}$ (by setting $Z=S^{0}$ in (1.0.2) and using associativity, we get that for all $s \in F\left(S^{0}\right), \mu_{X \wedge Y, S^{0}}\left(\mu_{X, Y}\left(\mathbf{1}_{X}, \mathbf{1}_{Y}\right), s\right)=$ $\mu_{X \wedge Y, S^{0}}\left(\mathbf{1}_{X \wedge Y}, s\right)$, and by the nondegeneracy (1.0.1) and naturality with respect to coordinate permutations, this implies the desired property). 
A left module over an FSP $F$ is a functor with stabilization $T$ with a natural transformation

$$
\ell_{X, Y}: F(X) \wedge T(Y) \rightarrow T(X \wedge Y)
$$

where the stabilization $\lambda_{X, Y}$ of $T$ is again given by left multiplication with $\mathbf{1}_{X}$ and

$$
\ell_{X \wedge Y, Z}\left(\mu_{X, Y} \wedge \operatorname{id}_{T(Z)}\right)=\ell_{X, Y \wedge Z}\left(\operatorname{id}_{F(X)} \wedge \ell_{Y, Z}\right)
$$

A right module over an FSP $F$ is defined analogously, with a natural transformation

$$
r_{X, Y}: T(X) \wedge F(Y) \rightarrow T(X \wedge Y)
$$

where the stabilization $\lambda_{X, Y}$ is given by right multiplication by the unit conjugated by the twist map $\tau$,

$$
\lambda_{X, Y}=T\left(\tau_{X, Y}\right) \circ r_{Y, X} \circ\left(\operatorname{id}_{T(Y)} \wedge \mathbf{1}_{X}\right) \circ \tau_{X, T(Y)}
$$

An $F$-bimodule over $F$ is both a right and a left module, where the action on the two sides commutes.

Given an FSP $A$ and $A$-bimodules $M_{1}, M_{2}, \ldots, M_{n}$, we define an $n$-simplicial functor with stabilization given in multidegree $\vec{k}=\left(k_{1}, k_{2}, \ldots, k_{n}\right)$ by

$$
U^{n}\left(A ; M_{1}, \ldots, M_{n}\right)_{\vec{k}}=\operatorname{holim}_{\underline{X} \in I^{\vec{k}+n}} \underline{\operatorname{Map}}\left(S^{\sqcup \underline{X}}, V^{n}(\underline{X})\right) .
$$

Here $I$ is the category whose objects correspond to the natural numbers and whose morphisms from $m$ to $n$ correspond to injections from $\{1,2, \ldots m\}$ to $\{1,2, \ldots n\}$. We write

$$
I^{\vec{k}+n}=I^{k_{1}+1} \times \cdots \times I^{k_{n}+1} .
$$

We let $\underline{\operatorname{Map}}(S, T)$ denote the functor with stabilization sending $Y \mapsto \operatorname{Map}(S, Y \wedge T)$, and for any $\underline{X}=\left(X^{1,0}, X^{1,1}, \ldots, X^{1, k_{1}}, \ldots, X^{n, 0}, \ldots, X^{n, k_{n}}\right) \in I^{\vec{k}+n}$,

$$
S^{\sqcup \underline{X}}=S^{X^{1,0}} \wedge S^{X^{1,1}} \wedge \cdots \wedge S^{X^{1, k_{1}}} \wedge \cdots \wedge S^{X^{n, 0}} \wedge \cdots \wedge S^{X^{n, k_{n}}}
$$

and

$$
\begin{aligned}
V^{n}(\underline{X}) & =M_{1}\left(S^{X^{1,0}}\right) \wedge A\left(S^{X^{1,1}}\right) \wedge \cdots \wedge A\left(S^{X^{1, k_{1}}}\right) \\
& \wedge M_{2}\left(S^{X^{2,0}}\right) \wedge A\left(S^{X^{2,1}}\right) \wedge \cdots \wedge A\left(S^{X^{2, k_{2}}}\right) \wedge \cdots \\
\cdots & \wedge M_{n}\left(S^{X^{n, 0}}\right) \wedge A\left(S^{X^{n, 1}}\right) \wedge \cdots \wedge A\left(S^{X^{n, k_{n}}}\right) .
\end{aligned}
$$

For simplicity of notation, we will from now on omit writing that we are looking at the simplicial realizations of our functors with stabilization such as $A$ and $M$ above. We have the usual Hochschild-type differentials and degeneracies in each simplicial direction between the $U^{n}\left(A ; M_{1}, \ldots, M_{n}\right)_{\vec{k}}$ for different $\vec{k}$ 's. 
We then set $U^{n}(A ; M)$ to be the diagonal of the multisimplicial set $U^{n}(A ; M, \ldots, M)$. It has a natural $C_{n}$-action permuting the $n$ blocks of $k+1$ coordinates in simplicial dimension $k$. If $M=A, U^{n}(A ; A)$ is simply the $n^{\prime}$ th edgewise subdivision of $\operatorname{THH}(A)$ - see [1] and [3].

For an FSP $A$, a left $A$-module $P$, and a right $A$-module $Q$, one can define a simplicial functor with stabilization $P \hat{\otimes}_{A} Q$, given in degree $k$ by

$$
\operatorname{holim}_{X \in I^{k+2}} \underline{\operatorname{Map}}\left(S^{\sqcup \underline{X}}, P\left(S^{X_{0}}\right) \wedge A\left(S^{X_{1}}\right) \wedge \cdots \wedge A\left(S^{X_{k}}\right) \wedge Q\left(S^{X_{k+1}}\right)\right)
$$

with the usual bar construction differentials (using $r_{X_{0}, X_{1}}$, the $\mu_{X_{i}, X_{i+1}}$, and $\ell_{X_{k}, X_{k+1}}$ ) and degeneracies (using $\mathbf{1}_{X_{i}}$ 's). For $m \mid n$, there is a $C_{n / m}$-equivariant simplicial homotopy equivalence

$$
U^{n}(A ; M) \simeq U^{n / m}\left(A ; M^{\hat{\otimes}_{A} m}\right)
$$

(see Lemmas 2.6 and 4.2 of [8]).

Definition (1.1) For $r \mid n$, we let

$$
\operatorname{Res}^{r}: U^{n}(A ; M)^{C_{n}} \rightarrow U^{n / r}(A ; M)^{C_{n / r}}
$$

be the restriction map obtained by observing that because of cofinality,

$$
U^{n}(A ; M)_{k} \simeq \operatorname{holim}_{\underline{X} \in I^{k+1}} \underline{\operatorname{Map}}\left(\left(S^{\sqcup \underline{X}}\right)^{\wedge n},\left(V^{1}(\underline{X})\right)^{\wedge n}\right),
$$

and restricting every map $f \in U^{n}(A ; M)_{k}^{C_{n}}$ to the $C_{r}$-fixed points of its domain,

$$
\left(\left(S^{\sqcup \underline{X}}\right)^{\wedge n}\right)^{C_{r}} \cong\left(S^{\sqcup \underline{X}}\right)^{\wedge(n / r)},
$$

which have to map to the fixed points in the range,

$$
\left(\left(V^{1}(\underline{X})\right)^{\wedge n}\right)^{C_{r}} \cong V^{1}(\underline{X})^{\wedge(n / r)},
$$

giving an element in

$$
\underset{\underline{X} \in I^{k+1}}{\operatorname{Map}}\left(\left(S^{\sqcup \underline{X}}\right)^{\wedge(n / r)}, V^{1}(\underline{X})^{\wedge(n / r)}\right)^{C_{n / r}} \simeq U^{n / r}(A ; M)^{C_{n / r}} .
$$

Let $\mathbb{N}^{\times}$denote the category of positive integers, with a morphism $n \rightarrow m$ whenever $m$ divides $n$. Then the $U^{n}(A ; M)^{C_{n}}$, equipped with the maps $\operatorname{Res}^{n / m}$, give a functor from $\mathbb{N}^{\times}$ to spectra, and we can look at its homotopy inverse limit over different full subcategories of $\mathbb{N}^{\times}$. 
Definition (1.2) Let

$$
\begin{aligned}
W(A ; M) & =\operatorname{holim}_{a \in \mathbb{N}^{\times}} U^{a}(A ; M)^{C_{a}} \\
W_{n}(A ; M) & =\operatorname{holim}_{a \in\{1,2, \ldots, n\}} U^{a}(A ; M)^{C_{a}} \\
W^{(p)}(A ; M) & =\operatorname{holim}_{\infty \leftarrow n} U^{p^{n}}(A ; M)^{C_{p^{n}}} .
\end{aligned}
$$

We will also often look at

$$
\underset{\infty \leftarrow n}{\operatorname{holim}} U^{t p^{n}}(A ; M)^{C_{p^{n}}} .
$$

As a spectrum, $\operatorname{holim}_{\infty \leftarrow n} U^{t p^{n}}(A ; M)^{C_{p^{n}}} \simeq W^{(p)}\left(A ; M^{\hat{\otimes}_{A} t}\right)$ because of (1.0.6), but it also comes with an action of $C_{t}$, since the restriction maps $\operatorname{Res}^{n / m}: U^{t p^{n}}(A ; M)^{C_{p^{n}}} \rightarrow$ $U^{t p^{m}}(A ; M)^{C_{p^{m}}}$ respect the quotient $C_{t}$ action.

Definition (1.3) Define (up to homotopy) Verschiebung maps

$$
v_{k}: U^{n}(A ; M)^{C_{n / k}} \rightarrow U^{n}(A ; M)^{C_{n}}
$$

using the fixed point transfer map. Recall that for a naive $G$-spectrum $X$ and a normal subgroup $H \triangleleft G$, the transfer $X^{H} \rightarrow X^{G}$ is induced on $G$-fixedpoints by the $G$-map

$$
G_{+} \wedge_{H} X \cong \bigvee_{G / H} X \rightarrow X
$$

where $G$ acts on $G_{+} \wedge_{H} X$ by left-multiplication on the first coordinate (and therefore $\left.\left(G_{+} \wedge_{H} X\right)^{G}=\left(\prod_{G / H} X\right)^{G}=X^{H}\right)$, and the map on the right is a $G$-equivariant folding map.

Note that for any $k \mid m$ and $m \mid n$, the diagram

$$
\begin{array}{rlr}
U^{n}(A ; M)^{C_{n / k}} & \stackrel{v_{k}}{\longrightarrow} & U^{n}(A ; M)^{C_{n}} \\
\downarrow \operatorname{Res}^{n / m} & & \downarrow \operatorname{Res}^{n / m} \\
U^{m}(A ; M)^{C_{m / k}} & \stackrel{v_{k}}{\longrightarrow} & U^{m}(A ; M)^{C_{m}}
\end{array}
$$

commutes, since the diagram

$$
\begin{array}{ccc}
\left(C_{n}\right)_{+} \wedge_{C_{k}} U^{n}(A ; M) & \stackrel{v_{k}}{\longrightarrow} & U^{n}(A ; M) \\
\downarrow \operatorname{Res}^{n / m} & & \downarrow \operatorname{Res}^{n / m} \\
\left(C_{m}\right)_{+} \wedge_{C_{k}} U^{m}(A ; M) & \stackrel{v_{k}}{\longrightarrow} & U^{m}(A ; M)
\end{array}
$$

commutes when we use the maps (1.3.1) as the horizontal maps. This means that we also get Verschiebung maps

$$
v_{p^{j}}: \operatorname{holim}_{\infty \leftarrow n} U^{p^{n+j}}(A ; M)^{C_{p^{n}}} \rightarrow W^{(p)}(A ; M) .
$$




\section{§2. A Sum Formula for $W$}

In this section, we will look at $W\left(A ; M_{1} \vee M_{2} \vee \cdots \vee M_{k}\right)$ and its variants, where $A$ is an FSP and $M_{1}, M_{2}, \ldots M_{k}$ (and therefore also $M_{1} \vee M_{2} \vee \cdots \vee M_{k}$ ) are $A$-bimodules. The result is that $W\left(A ; M_{1} \vee M_{2} \vee \cdots \vee M_{k}\right)$ is an (infinite, if $k>1$ ) product of $W$ of $A$ with coefficients in various tensor products of the $M_{i}$. We will need

Definition (2.1) For a pointed simplicial set $X$, we set

$$
w_{n}(X)=\bigwedge_{i=1}^{n} X / \bigcup_{\substack{a \mid n \\ a \neq n}}^{a} \bigwedge_{j=1}^{a} X
$$

where for each $a \mid n, a \neq n, \bigwedge_{j=1}^{a} X$ is viewed as a subset of $\bigwedge_{i=1}^{n} X$ via the diagonal map $\left(x_{1}, \ldots, x_{a}\right) \mapsto\left(x_{1}, \ldots, x_{a}, x_{1}, \ldots, x_{a}, \ldots, x_{1}, \ldots, x_{a}\right)$. In other words, $w_{n}(X)$ consists of the basepoint and all the non-periodic words of length $n$ consisting of points of $X$ other than the basepoint. It has a free (in the based sense) $C_{n}$ action induced from the $C_{n}$ action on $\bigwedge_{i=1}^{n} X$, and is in fact the largest quotient on which $C_{n}$ acts freely (the quotient by the singular set for this action).

In this section, we will often use $\tilde{w}_{n}(\underline{k})=w_{n}(\underline{k}) \backslash *$, where $\underline{k}=\{*, 1, \ldots, k\}$ for $k \geq 0$ : the set of all non-periodic words of length $n$ in $\{1, \ldots, k\}$.

Theorem (2.2) For any FSP $A$ and A-bimodules $M_{1}, \ldots, M_{k}$, we have natural (as functors of ordered $k$-tuples of $A$-bimodules) equivalences

$$
\begin{aligned}
W\left(A ; M_{1} \vee \cdots \vee M_{k}\right) & \simeq \prod_{a=1}^{\infty} \prod_{[f] \in \tilde{w}_{a}(\underline{\underline{k}}) / C_{a}} W\left(A ; M_{f(1)} \hat{\otimes}_{A} \cdots \hat{\otimes}_{A} M_{f(a)}\right) \\
W_{n}\left(A ; M_{1} \vee \cdots \vee M_{k}\right) & \simeq \prod_{a=1}^{n} \prod_{[f] \in \tilde{w}_{a}(\underline{\underline{k}}) / C_{a}} W_{\lfloor n / a\rfloor}\left(A ; M_{f(1)} \hat{\otimes}_{A} \cdots \hat{\otimes}_{A} M_{f(a)}\right) \\
W^{(p)}\left(A ; M_{1} \vee \cdots \vee M_{k}\right) & \simeq \prod_{b=0}^{\infty} \prod_{[f] \in \tilde{w}_{p^{b}}(\underline{k}) / C_{p^{b}}} W^{(p)}\left(A ; M_{f(1)} \hat{\otimes}_{A} \cdots \hat{\otimes}_{A} M_{f\left(p^{b}\right)}\right) .
\end{aligned}
$$

Proof. We use the fact that for any spaces $S, T$ and finite dimensional space $X$, there is a natural equivalence between the spectra associated to the functors with stabilization $\operatorname{Map}(X, S \vee T)$ and $\operatorname{Map}(X, S) \vee \operatorname{Map}(X, T)$, which is $G$-equivariant for any $G$ actions on $\overline{X, S}$, and $T$ (where $\bar{G}$ does not act on the argument of the functor with stabilization). We get that for any $n$ and $r$,

$$
\begin{aligned}
& U^{n}\left(A ; M_{1} \vee \cdots \vee M_{k}\right)_{r} \\
& \simeq \underset{\underline{X} \in I^{r+1}}{\operatorname{hocolim}} \underline{\operatorname{Map}}\left(\left(S^{\sqcup \underline{X}}\right)^{\wedge n},\left(\left(M_{1}\left(S^{X_{0}}\right) \vee \cdots \vee M_{k}\left(S^{X_{0}}\right)\right) \wedge A\left(S^{X_{1}}\right) \wedge \cdots \wedge A\left(S^{X_{r}}\right)\right)^{\wedge n}\right) \\
& \simeq \bigvee_{f:\{1, \ldots, n\} \rightarrow\{1, \ldots, k\}} \bigvee_{\underline{X} \in I^{r+1}}^{\operatorname{hocolim} \operatorname{Map}}\left(\left(S^{\sqcup \underline{X}}\right)^{\wedge n}, \bigwedge_{j=1}^{n}\left(M_{f(j)}\left(S^{X_{0}}\right) \wedge A\left(S^{X_{1}}\right) \wedge \cdots \wedge A\left(S^{X_{r}}\right)\right)\right) .
\end{aligned}
$$


Let

$$
U_{f, r}=\underset{\underline{X} \in I^{r+1}}{\operatorname{hocolim}} \underline{\operatorname{Map}}\left(\left(S^{\sqcup \underline{X}}\right)^{\wedge n}, \bigwedge_{j=1}^{n}\left(M_{f(j)}\left(S^{X_{0}}\right) \wedge A\left(S^{X_{1}}\right) \wedge \cdots \wedge A\left(S^{X_{r}}\right)\right)\right) .
$$

We can divide (2.2.1) according to the length of the period of the function $f$ (if $f$ is non-periodic, we regard its period as $n)$. Let $\tau$ be the $n$-cycle $(1,2, \ldots, n)$. Then $\tau$ acts on $U^{n}$ by sending $U_{f, r}$ to $U_{f \circ \tau^{-1}, r}$. We have

$$
\begin{aligned}
& \left(U^{n}\left(A ; M_{1} \vee \cdots \vee M_{k}\right)_{r}\right)^{C_{n}} \simeq\left(\bigvee_{a \mid n} \bigvee_{f \in \tilde{w}_{a}(\underline{k}) / C_{a}} \bigvee_{i=0}^{a-1} U_{f \circ \tau^{i}, r}\right)^{C_{n}} \\
& \quad \simeq\left(\bigvee_{a \mid n} \bigvee_{f \in \tilde{w}_{a}(\underline{k}) / C_{a}} \prod_{i=0}^{a-1} U_{f \circ \tau^{i}, r}\right)^{C_{n}} \simeq \bigvee_{a \mid n} \bigvee_{f \in \tilde{w}_{a}(\underline{k}) / C_{a}}\left(\prod_{i=0}^{a-1} U_{f \circ \tau^{i}, r}\right)^{C_{n}} \\
& \quad \simeq \bigvee_{a \mid n} \bigvee_{f \in \tilde{w}_{a}(\underline{k}) / C_{a}} U_{f \circ \tau^{i}, r}^{C_{n / a}}
\end{aligned}
$$

But if $f \in \tilde{w}_{a}(\underline{k}), U_{f, r}$ is homotopy equivalent to $U^{n}\left(A ; M_{f(1)}, M_{f(2)}, \ldots, M_{f(n)}\right)_{r, r, \ldots, r}$. So after taking geometric realization, we get

$$
\begin{aligned}
& U^{n}\left(A ; M_{1} \vee \cdots \vee M_{k}\right)^{C_{n}} \simeq \bigvee_{a \mid n} \bigvee_{f \in \tilde{w}_{a}(\underline{\underline{k}}) / C_{a}} U^{n}\left(A ; M_{f(1)}, \ldots, M_{f(a)}, \ldots, M_{f(1)}, \ldots, M_{f(a)}\right)^{C_{n / a}} \\
& \quad \simeq \bigvee_{a \mid n} \bigvee_{f \in \tilde{w}_{a}(\underline{\underline{k}}) / C_{a}} U^{n / a}\left(A ; M_{f(1)} \hat{\otimes}_{A} M_{f(2)} \hat{\otimes}_{A} \cdots \hat{\otimes}_{A} M_{f(a)}\right)^{C_{n / a}}
\end{aligned}
$$

where the last homotopy equivalence is based on an argument analogous to (1.0.6). We can convert (2.2.2) to a product, rather than a wedge, of the spectra in question before we take a homotopy inverse limit over Res. The proof of the theorem is completed by observing that for $m \mid n$,

$$
\operatorname{Res}^{n / m}: U^{n}\left(A ; M_{1} \vee \cdots \vee M_{k}\right)^{C_{n}} \rightarrow U^{m}\left(A ; M_{1} \vee \cdots \vee M_{k}\right)^{C_{m}}
$$

sends the factor corresponding to $f \in \tilde{w}_{a}(\underline{k}) / C_{a}$ to the factor corresponding to the same $f$ if $a \mid m$ (and otherwise to the basepoint), via

$$
\begin{aligned}
\operatorname{Res}^{n / m}: U^{n / a}\left(A ; M_{f(1)} \hat{\otimes}_{A}\right. & \left.M_{f(2)} \hat{\otimes}_{A} \cdots \hat{\otimes}_{A} M_{f(a)}\right)^{C_{n / a}} \\
& \rightarrow U^{m / a}\left(A ; M_{f(1)} \hat{\otimes}_{A} M_{f(2)} \hat{\otimes}_{A} \cdots \hat{\otimes}_{A} M_{f(a)}\right)^{C_{m / a}}
\end{aligned}
$$

since the domain of the restriction of a map is contained in the domain of the original map.

Applying (2.2) to the case $M_{1}=M_{2}=\cdots=M_{k}=A$, we get 
Application (2.3) For any FSP A,

$$
W\left(A, \bigoplus_{i=1}^{k} A\right) \simeq \prod_{a=1}^{\infty} \prod_{f \in \tilde{w}_{a}(\underline{\underline{k}}) / C_{a}} W(A ; A) .
$$

Proof. The inclusion $\vee_{i=1}^{k} A \hookrightarrow \bigoplus_{i=1}^{k} A$ of functors with stabilization becomes increasingly connected as it is evaluated on increasingly connected spaces, so $U^{n}(A ;-)^{C_{n}}$ and hence also the $W(A ;-)$ agree up to homotopy for these functors.

The multiplication map $A \hat{\otimes}_{A} A \rightarrow A$ is a homotopy equivalence (the former being a two-sided bar construction of $A$ with itself over itself), so $A^{\hat{\otimes}_{A} a} \simeq A$ as well.

Corollary (2.4) If $A$ is the FSP associated to a perfect field with positive characteristic, $W\left(A, \bigoplus_{i=1}^{k} A\right)$ is the Eilenberg-Mac Lane spectrum of the product of a countable number of copies of the Witt vectors of the field (in dimension 0 ) indexed by all the finite non-repeating words in $\{1, \ldots, k\}$.

Proof. Theorem 5.5, p. 72 in [6].

Corollary (2.5) If $A$ is the FSP associated to any commutative ring, $\pi_{0}\left(W\left(A, \bigoplus_{i=1}^{k} A\right)\right)$ is the product of a countable number of copies of the Witt vectors of the ring, indexed by all the finite non-repeating words in $\{1, \ldots, k\}$.

Proof. Addendum 3.3, p. 55 in [6].

Remark (2.6) Theorem (2.2) is also true if we use the definition of $U$ for functors with stabilization over a category, as in [8] - the same proof, with the obvious technical adjustments, works.

\section{$\S 3$. Splitting Formulae for $W$}

From its definition, $W(A ; M)$ is the homotopy inverse limit of the tower formed by $W_{n}(A ; M)$, with the maps $W_{n}(A ; M) \stackrel{\text { res }}{\longrightarrow} W_{n-1}(A ; M)$ induced on homotopy limits by restriction from the full subcategory on $\{1,2, \ldots, n\}$ to that on $\{1,2, \ldots, n-1\}$. We know from [8] (the proof will be reviewed in the proof of Theorem 3.2 below) that the layers in this tower are

$$
\operatorname{hofib}\left(W_{n}(A ; M) \stackrel{\text { res }}{\longrightarrow} W_{n-1}(A ; M)\right) \simeq U^{n}(A ; M)_{h C_{n}} .
$$

In the case $M=N \otimes X$, the $W_{n}$ correspond to the $n$ 'th stage Taylor approximations, and the layers can be thought of as the homogenous degree $n$ part of $W(A ; N \otimes X) \simeq$ $\tilde{K}(A ; N \otimes X)$.

In this section we will see how the layers fit together. If all nonzero integers act invertibly on the homotopy groups of $W(A ; M)$, it breaks up as the product of its layers. If we complete at some prime $p$, the layers are joined only if they have the same prime-to- $p$ part. The latter is the analog for $W(A ; M)$ of the splitting at $p$ of the big Witt vectors into a product of the $p$-Witt vectors, known classically in the algebraic setting and for $W(A ; A)$ by $[6]$. We first need a 
Lemma (3.1) If $j$ is a (naive) $G$-spectrum for some finite group $G$ so that multiplication by $|G|$ acts invertibly on $\pi_{*}(\underline{j})$ then the norm map induces an equivalence

$$
(\underline{j})_{h G} \rightarrow(\underline{j})^{h G} .
$$

Proof. To define the norm map, consider the spectrum $\bigvee_{g \in G} \underline{j}$. The product $G_{1} \times G_{2}$, where $G_{1}=G_{2}=G$, acts on it as follows: $G_{1}$ permutes the copies of $\underline{j}$, by having $g \in G_{1}$ send the $h$ 'th copy of $j$ to the $h g^{-1}$ 'th copy; $G_{2}$ permutes the copies and also acts on them, so $g \in G_{2}$ sends the $\bar{h}^{\prime}$ th copy $j$ to the $g h^{\prime}$ 'th copy while also acting on it on the left (a diagonal action on $G_{+} \wedge \underline{j}$ ). The norm map can now be described as the canonical map

$$
\underline{j}_{h G} \simeq\left(\left(\bigvee_{g \in G} \underline{j}\right)^{h G_{1}}\right)_{h G_{2}} \rightarrow\left(\left(\bigvee_{g \in G} \underline{j}_{h G_{2}}\right)^{h G_{1}} \simeq \underline{j}^{h G}\right.
$$

Note that if the order of $G$ acts invertibly on $\pi_{*}(j)$, the $E_{2}$ spectral sequence

$$
H_{p}\left(G, \pi_{q}(\underline{j})\right) \Rightarrow \pi_{p+q}\left(\underline{j}_{h G}\right)
$$

in the right half-plane coming from the simplicial filtration on $E G$ vanishes except for $\pi_{*}(j)_{G}$ in the 0'th column. Similarly, the $E_{2}$ spectral sequence

$$
H^{-p}\left(G, \pi_{q}(\underline{j})\right)
$$

in the left half-plane vanishes except for $\pi_{*}(\underline{j})^{G}$ in the 0 'th column, and therefore converges, and converges to $\pi_{*}\left(j^{h G}\right)$.

Once we know the homotopy groups of the terms involved, we can study the map the norm induces on them. It is the canonical change-of-order map

$$
\pi_{*}(\underline{j})_{G} \cong\left(\left(\bigoplus_{g \in G} \pi_{*}(\underline{j})\right)^{G_{1}}\right)_{G_{2}} \rightarrow\left(\left(\bigoplus_{g \in G} \pi_{*}(\underline{j})\right)_{G_{2}}\right)^{G_{1}} \cong \pi_{*}(\underline{j})^{G}
$$

sending $[a] \mapsto \sum_{g \in G} g a$. When multiplication by $|G|$ is invertible on $\pi_{*}(\underline{j})$, this map is an isomorphism (injective because for all $a \in \pi_{*}(\underline{j}),[|G| \cdot a]=\left[\sum_{g \in G} g a\right]$ in $\pi_{*}(\underline{j})_{G}$, surjective because for all $\left.a \in \pi_{*}(\underline{j})^{G}, \sum_{g \in G} g a=|G| \cdot a\right)$.

Theorem (3.2) Let $A$ be an FSP and $M$ an A-bimodule. Assume that multiplication by $n$ acts invertibly on the homotopy groups of $U^{a}(A ; M)$ for all a|n. Then the map

$$
U^{n}(A ; M)^{C_{n}} \stackrel{\phi_{n}}{\longrightarrow} \prod_{a \mid n} U^{a}(A ; M)^{h C_{a}}
$$

given by a product of the maps

$$
U^{n}(A ; M)^{C_{n}} \stackrel{\operatorname{Res}^{n / a}}{\longrightarrow} U^{a}(A ; M)^{C_{a}} \stackrel{\text { inc }}{\hookrightarrow} U^{a}(A ; M)^{h C_{a}}
$$


(where the inclusion is the standard inclusion of fixed points into homotopy fixed points) is an equivalence. Therefore, if all $1 \leq n \leq n_{0}$ act invertibly on $\pi_{*}(M)$ (and therefore on all the $\left.\pi_{*}\left(U^{a}(A ; M)\right)\right)$, we have an equivalence

$$
W_{n_{0}}(A ; M) \stackrel{\sim}{\longrightarrow} \prod_{a=1}^{n_{0}} U^{a}(A ; M)^{h C_{a}}
$$

and if $\pi_{*}(M)$ is a vector space over $\mathbb{Q}$ (and therefore all the $\pi_{*}\left(U^{a}(A ; M)\right)$ are, too), we have an equivalence

$$
W(A ; M) \stackrel{\sim}{\longrightarrow} \prod_{a \in \mathbb{N}^{\times}} U^{a}(A ; M)^{h C_{a}} .
$$

Proof. Since for $a \mid m$ and $m \mid n, \operatorname{Res}^{n / a} \simeq \operatorname{Res}^{m / a} \circ \operatorname{Res}^{n / m}$, the results for $W_{n_{0}}(A ; M)$ and $W(A ; M)$ follow from the result for $U^{n}(A ; M)^{C_{n}}$ by taking homotopy inverse limits over the categories $\left\{1, \ldots, n_{0}\right\}$ and $\mathbb{N}^{\times}$, respectively.

We will use induction on $n$ to show that if $n$ acts invertibly on $\pi_{*}\left(U^{a}(A ; M)\right)$ for all $a \mid n$, the map $\phi_{n}$ is an equivalence. For $n=1$, there is nothing to show. For $n>1$, we look at the diagram

$$
\begin{aligned}
& U^{n}(A ; M)_{h C_{n}} \stackrel{i}{\longrightarrow} \quad U^{n}(A ; M)^{C_{n}} \quad \stackrel{\text { res }}{\longrightarrow} \operatorname{holim}_{a \mid n, a \neq n} U^{a}(A ; M)^{C_{a}} \\
& \downarrow \phi_{n} \quad \downarrow \operatorname{holim}_{a \mid n, a \neq n} \phi_{a} \\
& U^{n}(A ; M)^{h C_{n}} \longrightarrow \prod_{a \mid n} U^{a}(A ; M)^{h C_{a}} \longrightarrow \prod_{a \mid n, a \neq n} U^{a}(A ; M)^{h C_{a}} .
\end{aligned}
$$

The top row is the fibration of Proposition 5.6 in [8], whose proof will be reviewed below. The map res comes from restricting categories in the homotopy inverse limit from all of the divisors of $n$ to all of its divisors other than itself, since $\operatorname{holim}_{a \mid n} U^{a}(A ; M)^{C_{a}} \simeq$ $U^{n}(A ; M)^{C_{n}}$. The bottom row is the obvious product fibration. It is also what we get by applying the same category restriction to the homotopy inverse limit of the functor $n \mapsto \prod_{a \mid n} U^{a}(A ; M)^{h C_{a}}$ as we did to the functor $n \mapsto U^{n}(A ; M)^{C_{n}}$ in the top row, which is why the diagram homotopy-commutes. In fact, it is strictly commutative: in the bottom row, the homotopy inverse limit agrees with the inverse limit. So if we replaced the rightmost term of the top row by $\lim _{a \mid n, a \neq n} U^{a}(A ; M)^{C_{a}}$, the composition with res would not change and we get

$$
\underset{a \mid n, a \neq n}{\operatorname{holim}} \phi_{a} \circ \operatorname{res}=\lim _{a \mid n, a \neq n} \phi_{a} \circ \operatorname{res}=\prod_{a \mid n, a \neq n} \operatorname{inc} \circ \operatorname{Res}^{n / a} .
$$

By the inductive hypothesis, which we use only for the proper divisors of $n$, the rightmost vertical map is an equivalence (since if $n$ acts invertibly on the homotopy, all its proper divisors do too). But since the diagram commutes, the map $\phi_{n} \circ i$ factors through a map

$$
U^{n}(A ; M)_{h C_{n}} \stackrel{i_{n}}{\longrightarrow} U^{n}(A ; M)^{h C_{n}}
$$

which is equal to the composition

$$
U^{n}(A ; M)_{h C_{n}} \stackrel{i}{\longrightarrow} U^{n}(A ; M)^{C_{n}} \hookrightarrow U^{n}(A ; M)^{h C_{n}} .
$$


The map $i_{n}$ is the norm of Lemma (3.1) so since multiplication by $n$ is invertible, it is invertible. To see that $i_{n}$ is the norm, recall from Proposition 5.6 in [8] the proof that

$$
U^{n}(A ; M)_{h C_{n}} \simeq \operatorname{hofib}\left(U^{n}(A ; M)^{C_{n}} \stackrel{\text { res }}{\longrightarrow} \operatorname{holim}_{a \mid n, a \neq n} U^{a}(A ; M)^{C_{a}}\right) .
$$

It observes that levelwise, the homotopy fiber in question can be viewed as a homotopy direct limit (over $\underline{X}$ ) of the total homotopy fiber of

$$
\underline{\operatorname{Map}}\left(\left(S^{\sqcup \underline{X}}\right)^{\wedge n}, V^{1}(\underline{X})^{\wedge n}\right)^{C_{n}}
$$

mapping into

$$
\underline{\operatorname{Map}}\left(\left(\left(S^{\sqcup \underline{X}}\right)^{\wedge n}\right)^{C_{n / a}}, V^{1}(\underline{X})^{\wedge n}\right)^{C_{n}}
$$

over all $a \mid n, a \neq n$, where $V^{1}(\underline{X})$ is as in (1.0.4). This total homotopy fiber is equivalent to $\operatorname{Map}\left(\mathcal{U}, V^{1}(\underline{X})^{\wedge n}\right)^{C_{n}}$ where $\mathcal{U}$ is the total cofiber of the inclusions

$$
\left(\left(S^{\sqcup \underline{X}}\right)^{\wedge n}\right)^{C_{n / a}} \hookrightarrow\left(S^{\sqcup \underline{X}}\right)^{\wedge n}
$$

for all $a \mid n, a \neq n$. We can write $\mathcal{U}=\left(S^{\sqcup \underline{X}}\right)^{\wedge n} / \bigcup_{\substack{a \mid n \\ a \neq n}}\left(\left(S^{\sqcup} \underline{X}\right)^{\wedge n}\right)^{C_{n / a}}$ (replacing the total homotopy cofiber by the quotient since the inclusions in question are cofibrations). Then, since $C_{n}$ acts freely (in the pointed sense) on $\mathcal{U}$, the obvious inclusion induces an equivalence

$$
\underline{\operatorname{Map}}\left(\mathcal{U}, V^{1}(\underline{X})^{\wedge n}\right)^{C_{n}} \simeq \underline{\operatorname{Map}}\left(\mathcal{U}, V^{1}(\underline{X})^{\wedge n}\right)^{h C_{n}}
$$

Since $\mathcal{U}$ is a free (in the pointed sense) complex of dimension one higher than the connectedness of $V^{1}(\underline{X})^{\wedge n}$, by work of Goodwillie (described in Theorem 5.5 of [8]) the Tate, or norm map

$$
\underline{\operatorname{Map}}\left(\mathcal{U}, V^{1}(\underline{X})^{\wedge n}\right)^{h C_{n}} \simeq \underline{\operatorname{Map}}\left(\mathcal{U}, V^{1}(\underline{X})^{\wedge n}\right)_{h C_{n}}
$$

is an equivalence (in our case where $n$ acts invertibly on the homotopy groups, this is of course also true by Lemma (3.1)). Now $V^{1}(\underline{X})$ is $|\underline{X}|$-connected, so for connectivity reasons the collapse map $\left(S^{\sqcup \underline{X}}\right)^{\wedge n} \stackrel{\mathcal{C}}{\rightarrow} \mathcal{U}$ induces an equivalence

$$
\underline{\operatorname{Map}}\left(\mathcal{U}, V^{1}(\underline{X})^{\wedge n}\right) \simeq \underline{\operatorname{Map}}\left(\left(S^{\sqcup \underline{X}}\right)^{\wedge n}, V^{1}(\underline{X})^{\wedge n}\right)
$$

which respects the $C_{n}$-action and so induces an equivalence on homotopy fixedpoints and homotopy quotients. We have

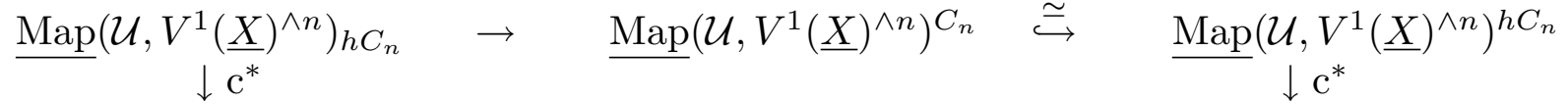

$$
\begin{aligned}
& \underline{\operatorname{Map}}\left(\left(S^{\sqcup \underline{X}}\right)^{\wedge n}, V^{1}(\underline{X})^{\wedge n}\right)_{h C_{n}} \rightarrow \underline{\operatorname{Map}}\left(\left(S^{\sqcup \underline{X}}\right)^{\wedge n}, V^{1}(\underline{X})^{\wedge n}\right)^{C_{n}} \hookrightarrow \underline{\operatorname{Map}}\left(\left(S^{\sqcup \underline{X}}\right)^{\wedge n}, V^{1}(\underline{X})^{\wedge n}\right)^{h C_{n}}
\end{aligned}
$$


where the composition of the maps in the first row (equivalences by (3.2.2) and (3.2.3)) is the norm. The vertical maps are equivalences by (3.2.4). The bottom row is the composition $i_{n}$ we are interested in, but we could have written the norm there and the diagram would still homotopy-commute. Since all edges of the rectangle except for the bottom one are homotopy equivalences, this means that up to homotopy, $i_{n}$ is the norm map, which is what we needed to show. In fact it shows that for every $\underline{X}$, and therefore by taking the homotopy direct limit in each simplicial degree, $i_{n}$ is a homotopy equivalence. But taking homotopy fixedpoints does not in general commute with realizations so it does not show that $i_{n}$ would be an equivalence without the invertibility of $n$.

Since the norm map is an equivalence if $\pi_{*}(M)$ is a vector space over $\mathbb{Q}$, we can write the last decomposition of Theorem (3.2) as

$$
W(A ; M) \simeq \prod_{a \in \mathbb{N}^{\times}} U^{a}(A ; M)_{h C_{a}} .
$$

When $A$ is the FSP associated to a discrete ring and $M$ is the functor with stabilization associated to a discrete $A$-bimodule, we can further describe these layers. We can define a multisimplicial set $U_{\text {lin }}^{a}(A ; M)$ with

$$
U_{\text {lin }}^{a}(A ; M)_{r_{1}, \ldots, r_{a}}=M \otimes A^{\otimes r_{1}} \otimes M \otimes A^{\otimes r_{2}} \otimes \cdots \otimes M \otimes A^{\otimes r_{a}} .
$$

Since rationally, an Eilenberg-Mac Lane spectrum $H A$ of a discrete ring $A$ has no homotopy above dimension zero, the collapse map $H A \wedge H B \rightarrow H(A \otimes B)$ is a rational equivalence, and the realizations

$$
U^{a}(A ; M) \simeq_{\mathbb{Q}} U_{\text {lin }}^{a}(A ; M)
$$

(where the latter is a spectrum because of its simplicial abelian group structure). So we have

$$
U^{a}(A ; M)_{h C_{a}} \simeq_{\mathbb{Q}} U_{\operatorname{lin}}^{a}(A ; M)_{h C_{a}} .
$$

As before, if $\pi_{*}(X)$ is a $\mathbb{Q}$-vector space, since $\mathbb{Q}$ with the trivial action is a projective $\mathbb{Q}\left[C_{a}\right]$-module, the terms in the spectral sequence

$$
H_{p}\left(C_{a} ; \pi_{q}(X)\right) \Rightarrow \pi_{p+q}\left(X_{h C_{a}}\right) .
$$

vanish unless $p=0$, and $\pi_{q}\left(X_{h C_{a}}\right) \cong \pi_{q}(X) / C_{a}$. Since $\pi_{*}\left(U_{\operatorname{lin}}^{a}(A ; M)\right) \cong \operatorname{HH}_{*}\left(A ; M^{\hat{\otimes}_{A} a}\right)$, we can (if $\pi_{*}(M)$ is a $\mathbb{Q}$-vector space) deduce that

$$
\pi_{*}\left(U^{a}(A ; M)_{h C_{a}}\right) \cong \operatorname{HH}_{*}\left(A ; M^{\hat{\otimes}_{A} a}\right) / C_{a}
$$

and write out the formula

$$
\pi_{*}(W(A ; M)) \cong \prod_{a=1}^{\infty} \operatorname{HH}_{*}\left(A ; M^{\hat{\otimes}_{A} a}\right) / C_{a} .
$$

If we make some further assumptions, we can understand the $C_{a}$-quotient on the right in terms of the obvious $C_{a^{-}}$action on $M^{\hat{\otimes}_{A} a}$. 
Claim (3.3) If $A$ is a commutative ring and $M$ a symmetric, flat A-bimodule whose homotopy groups are vector spaces over $\mathbb{Q}$, then $W(A ; M)$ is equivalent to a product of Eilenberg-Mac Lane spectra, and

$$
\pi_{*}(W(A ; M)) \cong \prod_{a=1}^{\infty}\left(\mathrm{HH}_{*}(A) \otimes_{A}\left(M^{\otimes_{A} a} / C_{a}\right)\right)
$$

Proof. We can rewrite (the diagonal of) $U_{\text {lin }}^{a}$ as the simplicial abelian group

$$
i \mapsto\left(M \otimes A^{\otimes i}\right)^{\otimes a} \cong\left(A \otimes A^{\otimes i}\right)^{\otimes a} \otimes_{A^{\otimes a}} M^{\otimes a}
$$

since the elements of $A$ commute with everything. (Here $A^{\otimes a}$ acts on $\left(A \otimes A^{\otimes i}\right)^{\otimes a}$ by multiplying each $j$ 'th copy of $A$ into the leftmost $A$ of the $j^{\prime}$ th copy of $A \otimes A^{\otimes i}$.) The simplicial operators act only on $\left(A \otimes A^{\otimes i}\right)^{\otimes a}$, which is exactly the $a^{\prime}$ th edgewise subdivision (see [1]) of the standard Hochschild complex of $A$. Since $M$ is flat over $A, M^{\otimes a}$ is flat over $A^{\otimes a}$, so the homotopy of the realization of $U_{\operatorname{lin}}^{a}$ as given in (3.3.1) is isomorphic to

$$
\mathrm{HH}_{*}(A) \otimes_{A^{\otimes a}} M^{\otimes a} .
$$

Now the $C_{a}$ action on the $a^{\prime}$ th edgewise subdivision of the Hochschild complex, $i \mapsto$ $\left(A \otimes A^{\otimes i}\right)^{\otimes a}$, is non-trivial, but once we pass to homology it becomes trivial since it is an action on homotopy groups given by a $C_{a}$-action which is the restriction of an $S^{1}$-action (see [1]). This means that if we multiply $\operatorname{HH}_{*}(A)$ by $1 \otimes \alpha \otimes 1 \otimes \cdots \otimes 1$, we could without changing the result first rotate, then multiply by $\alpha$ on the left, and finally rotate back. So the $A^{\otimes a}$ action on $\mathrm{HH}_{*}(A)$ factors through $A$, and we can write

$$
\begin{aligned}
\mathrm{HH}_{*}(A) & \otimes_{A^{\otimes a}} M^{\otimes a} \cong \mathrm{HH}_{*}(A) \otimes_{A} A \otimes_{A^{\otimes a}} M^{\otimes a} \\
& \cong \mathrm{HH}_{*}(A) \otimes_{A}\left(A \otimes_{A^{\otimes a}} M^{\otimes a}\right) \cong \mathrm{HH}_{*}(A) \otimes_{A} M^{\otimes_{A} a}
\end{aligned}
$$

with the $C_{a}$-action concentrated in the second factor. Now the $C_{a}$-action on $U_{\text {lin }}^{a}$ preserves the simplicial abelian structure, so $\pi_{*}\left(U_{\operatorname{lin}}^{a} / C_{a}\right)$ is the homology of the quotient of the

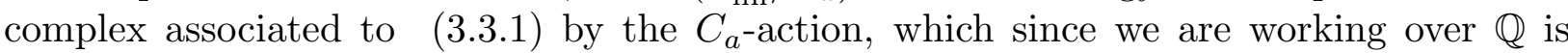
rationally isomorphic to $\mathrm{HH}_{*}(A) \otimes_{A} M^{\otimes_{A} a} / C_{a}$.

We are, however, interested in the torsion for algebraic K-theory and therefore also for $W$. If we want to look at $p$-torsion, we can complete at $p$, where we find that the layers $U^{n}(A ; M)_{h C_{n}}$ of $W(A ; M)$ glue according to their prime-to- $p$ part. The main result of this section is

Theorem (3.4) Let $A$ be an FSP, $M$ an A-bimodule, and $p$ a prime. Let $n=k p^{b}$, $(k, p)=1$. Then there is an equivalence

$$
\left(U^{n}(A ; M)^{C_{n}}\right)_{p}^{\wedge} \stackrel{r_{n}}{\longrightarrow} \prod_{\ell \mid k}\left(\left(U^{\ell p^{b}}(A ; M)^{C_{p^{b}}}\right)_{p}^{\wedge}\right)^{h C_{\ell}}
$$


given by a product of the maps

$$
\left(U^{n}(A ; M)^{C_{n}}\right)_{p}^{\wedge} \stackrel{\operatorname{Res}^{k / \ell}}{\longrightarrow}\left(U^{\ell p^{b}}(A ; M)^{C_{\ell p^{b}}}\right)_{p}^{\wedge} \underset{\mathrm{inc}}{\hookrightarrow}\left(\left(U^{\ell p^{b}}(A ; M)^{C_{p^{b}}}\right)_{p}^{\wedge}\right)^{h C_{\ell}}
$$

(where the map inc is induced by the standard inclusion of fixed points into homotopy fixed points; taking $C_{\ell}$ homotopy fixedpoints commutes with $p$ completion since $\left.(\ell, p)=1\right)$. This yields an equivalence

$$
\begin{aligned}
W(A ; M)_{p}^{\wedge} & \simeq \prod_{(k, p)=1} \operatorname{holim}_{\infty \leftarrow b}\left(\left(U^{k p^{b}}(A ; M)^{C_{p^{b}}}\right)_{p}^{\wedge}\right)^{h C_{k}} \\
& \simeq \prod_{(k, p)=1} \operatorname{holim}_{\infty \leftarrow b}\left(\left(U^{k p^{b}}(A ; M)^{C_{p^{b}}}\right)_{p}^{\wedge}\right)_{h C_{k}}
\end{aligned}
$$

Proof. For $n=k p^{b},(k, p)=1$, set $\Pi U(n)=\prod_{\ell \mid k}\left(U^{\ell p^{b}}(A ; M)^{C_{p^{b}}}\right)^{h C_{\ell}}$. For $m=\ell p^{a}, \ell \mid k$ and $a \leq b$, we let

$$
(\Pi \operatorname{Res})^{n / m}: \Pi U(n) \rightarrow \Pi U(m)
$$

be the map which sends each factor $\left(U^{j p^{b}}(A ; M)^{C_{p^{b}}}\right)^{h C_{j}}$ for $j \mid \ell$ to the corresponding factor in $\Pi U(m)$ by the map

$$
\left(\operatorname{Res}^{p^{b-a}}\right)^{h C_{j}}:\left(U^{j p^{b}}(A ; M)^{C_{p^{b}}}\right)^{h C_{j}} \rightarrow\left(U^{j p^{a}}(A ; M)^{C_{p^{a}}}\right)^{h C_{j}}
$$

and each factor $\left(U^{j p^{b}}(A ; M)^{C_{p^{b}}}\right)^{h C_{j}}$ for $j$ which does not divide $\ell$ to the basepoint. Then, since $\operatorname{Res}^{p^{b-a}} \circ \operatorname{Res}^{k / j} \simeq \operatorname{Res}^{\ell / j} \circ \operatorname{Res}^{p^{b-a} \cdot k / \ell}$, we have a commutative square

$$
\begin{array}{ccc}
U^{n}(A ; M)^{C_{n}} & \stackrel{r_{n}}{\longrightarrow} & \Pi U(n) \\
\downarrow & \downarrow \operatorname{Res}^{n / m} & \\
U^{m}(A ; M)^{C_{m}} & \stackrel{r_{m}}{\longrightarrow} & \Pi U(m) .
\end{array}
$$

We will prove by induction on $n$ that each $r_{n}$ induces an equivalence on $p$-completions. The result for $W(A ; M)_{p}^{\wedge}$ will follow from the result for all the $\left(U^{n}(A ; M)_{p}^{\wedge}\right)^{C_{n}}$ by taking a homotopy inverse limit over $\mathbb{N}^{\times}$of both sides. The two formulae for $W(A ; M)_{p}^{\wedge}$ agree by Lemma (3.1); we will find the version with homotopy quotients easier to calculate.

We have $n=k p^{b}$ with $(k, p)=1$. In the base case of the induction, the case $n=1$, there is nothing to show. For $n=k$ coprime to $p$, the map $r_{n}$ is exactly $\phi_{n}$ of Theorem (3.2), where it is proven that it is an equivalence if multiplication by $n$ is invertible on the homotopy groups of the spaces in question, here $U^{\ell}(A ; M)_{p}^{\wedge}$ for all $\ell \mid k$.

So we will assume that $n=k p^{b}$ with $b>0$ and that the result is known for all the proper divisors of $n$. Consider the diagram

$$
\begin{aligned}
& U^{k p^{b}}(A ; M)_{h C_{k p^{b}}} \quad \stackrel{i}{\longrightarrow} \quad U^{k p^{b}}(A ; M)^{C_{k p^{b}}} \quad \stackrel{\text { res }}{\longrightarrow} \operatorname{holim}{\substack{\ell p^{a} \mid k p^{b} \\
\ell p^{a} \neq k p^{b}}} U^{\ell p^{a}}(A ; M)^{C_{\ell p^{a}}} \\
& \downarrow r_{k p^{b}} \\
& \left(U^{k p^{b}}(A ; M)_{h C_{p^{b}}}\right)^{h C_{k}} \longrightarrow \prod_{\ell \mid k}\left(U^{\ell p^{b}}(A ; M)^{C_{p^{b}}}\right)^{h C_{\ell}} \stackrel{\mathrm{f}}{\longrightarrow} \prod_{\substack{\ell \mid k \\
\ell \neq k}}\left(U^{\ell p^{b}}(A ; M)^{C_{p^{b}}}\right)^{h C_{\ell}} \\
& \times\left(U^{k p^{b-1}}(A ; M)^{C_{p^{b-1}}}\right)^{h C_{k}}
\end{aligned}
$$


where top row is the fibration of Proposition 5.6 in [8], as reviewed in the proof of Theorem (3.2), and the map res comes from restricting the category over which we take a homotopy inverse limit from $\{m: m \mid n\}$ to $\{m: m \mid n, m \neq n\}$. On the bottom row, the map $\mathrm{f}$ is induced by the same restriction of categories when we take homotopy inverse limits of the functor $\Pi U$. It is the identity map on all factors except the factor corresponding to $k$, where it is

$$
\left(U^{k p^{b}}(A ; M)^{C_{p^{b}}}\right)^{h C_{k}} \stackrel{\left(\operatorname{Res}^{p}\right)^{h C_{k}}}{\longrightarrow}\left(U^{k p^{b-1}}(A ; M)^{C_{p^{b-1}}}\right)^{h C_{k}} .
$$

By the method of proof Proposition 5.6 in [8] again, we have a fibration

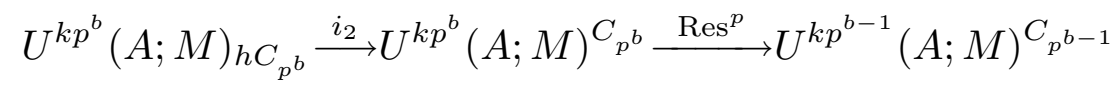

which explains why the fiber of $\mathrm{f}$ is $\left(U^{k p^{b}}(A ; M)_{h C_{p^{b}}}\right)^{h C_{k}}$.

The diagram (3.4.1) homotopy commutes since restricting the category over which we are taking a homotopy inverse limit commutes with applying the the transformation from $U^{t}(A ; M)^{C_{t}}$ to $\Pi U(t)$ for every $t$. Note that for $m \mid k, m \neq k$, the $m$ 'th coordinate in holim $r_{\ell p^{a}} \circ$ res is inc $\circ \operatorname{Res}^{k / m}$, just like the $m^{\prime}$ th coordinate in $r_{k p^{b}}$, so in all but the $k^{\prime}$ th coordinate, the diagram (3.4.1) actually commutes. So the map $r_{k p^{b}} \circ i$ factors through a map

$$
U^{k p^{b}}(A ; M)_{h C_{k p^{b}}} \rightarrow\left(U^{k p^{b}}(A ; M)_{h C_{p^{b}}}\right)^{h C_{k}}
$$

whose composition with the map $\left(\operatorname{Res}^{p}\right)^{h C_{k}}$ from (3.4.2) is nullhomotopic. We will show that this map, which could be added as a leftmost vertical map in diagram (3.4.1) while keeping the diagram homotopy commutative, is up to homotopy the $C_{k}$-norm map. Since $k$ is coprime to $p$, by Lemma (3.1) the norm is an equivalence on $p$ completions. This would finish the proof, since the rightmost vertical map in (3.4.1) is an equivalence by the inductive hypothesis.

Using the proof of Proposition 5.6 in [8] again, $U^{k p^{b}}(A ; M)_{h C_{k p^{b}}}$ is shown to be the fiber of res by explaining why levelwise (in each simplicial degree) $U^{k p^{b}}(A ; M)_{h C_{k p^{b}}}$ is homotopy equivalent to the homotopy limit over all $\underline{X}$ of $\operatorname{Map}\left(\mathcal{U}_{1}, V^{1}(\underline{X})^{\wedge k p^{b}}\right)^{C_{k p^{b}}}$, where $\mathcal{U}_{1}$ (like $\mathcal{U}$ in the proof of Theorem (3.2)) is the total cofiber of the inclusions $\left(\left(S^{\sqcup} \underline{X}\right)^{\wedge k p^{b}}\right)^{C_{m}} \hookrightarrow\left(S^{\sqcup} \underline{X}\right)^{\wedge k p^{b}}$ over all $m \mid k p^{b}, m \neq 1$. In these terms, the map $i$ in diagram (3.4.1) is induced by the collapse map $\left(S^{\sqcup \underline{X}}\right)^{\wedge n} \stackrel{\mathrm{c}_{1}}{\longrightarrow} \mathcal{U}_{1}$.

Similarly, the fiber in (3.4.3) should be thought of levelwise as the limit over $\underline{X}$ of $\operatorname{Map}\left(\mathcal{U}_{2}, V^{1}(\underline{X})^{\wedge k p^{b}}\right)^{C_{k p^{b}}}$ where $\mathcal{U}_{2}$ is the cofiber of the inclusion $\left(\left(S^{\sqcup \underline{X}}\right)^{\wedge k p^{b}}\right)^{C_{p}} \hookrightarrow$ $\left(S^{\sqcup \underline{X}}\right)^{\wedge} k p^{b}$, and $i_{2}$ is induced by the collapse map $\left(S^{\sqcup \underline{X}}\right)^{\wedge n} \stackrel{\mathrm{c}_{2}}{\longrightarrow} \mathcal{U}_{2}$. The second row in equation (3.4.1) has the $C_{k}$ homotopy fixed points of the fibration (3.4.3) in the factor corresponding to $k$.

With this notation, for each $\underline{X}$, the composites

$$
\underline{\operatorname{Map}}\left(\mathcal{U}_{1}, V^{1}(\underline{X})^{\wedge k p^{b}}\right)^{C_{k p^{b}} \hookrightarrow}\left(\underline{\operatorname{Map}}\left(\mathcal{U}_{1}, V^{1}(\underline{X})^{\wedge k p^{b}}\right)^{C_{p^{b}}}\right)^{h C_{k}} \stackrel{c_{3}^{*}}{\longrightarrow}\left(\underline{\operatorname{Map}}\left(\mathcal{U}_{2}, V^{1}(\underline{X})^{\wedge k p^{b}}\right)^{C_{p^{b}}}\right)^{h C_{k}}
$$


where $c_{3}: \mathcal{U}_{1} \rightarrow \mathcal{U}_{2}$ is the obvious collapse map $\left(c_{3} \circ c_{2}=c_{1}\right)$ induce the map we want which, if used between the leftmost terms of both rows, would make diagram (3.4.1) commute. The map inc in (3.4.5) is an equivalence by the freeness of the action of $C_{k}$ on $\mathcal{U}_{1}$, and both the first and the second term are, for that reason, equivalent to $\underline{\operatorname{Map}}\left(\mathcal{U}_{1}, V^{1}(\underline{X})^{\wedge k p^{b}}\right)_{h C_{k p^{b}}}$. We have a commutative diagram

$$
\begin{array}{ccc}
\underline{\operatorname{Map}}\left(\mathcal{U}_{1}, V^{1}(\underline{X})^{\wedge k p^{b}}\right)_{h C_{k p^{b}}} & \stackrel{c_{3}^{*}}{\downarrow} & \underline{\operatorname{Map}}\left(\mathcal{U}_{2}, V^{1}(\underline{X})^{\wedge k p^{b}}\right)_{h C_{k p^{b}}} \\
\left(\underline{\operatorname{Map}}\left(\mathcal{U}_{1}, V^{1}(\underline{X})^{\wedge k p^{b}}\right)_{h C_{p^{b}}}\right)^{h C_{k}} & \stackrel{c_{3}^{*}}{\longrightarrow} & \left(\underline{\operatorname{Map}}\left(\mathcal{U}_{2}, V^{1}(\underline{X})^{\wedge k p^{b}}\right)_{h C_{p^{b}}}\right)^{h C_{k}},
\end{array}
$$

where the vertical maps are the $C_{k}$-norms (and the left one is an equivalence even before completion), and the maps $c_{3}^{*}$ are homotopy equivalences because they are the maps induced on homotopy quotients and fixedpoints, respectively, by a homotopy equivalence which is a $C_{k p^{b}}$ map. The composition of (3.4.5) is, up to homotopy equivalence, the composition of maps from the top left to the bottom right of (3.4.6), but the only map in (3.4.6) which is not already a homotopy equivalence is the norm in the right column. Therefore the composition of $(3.4 .5)$ is, up to homotopy equivalence, the $C_{k}$ norm. Since the norm commutes with $p$-completion, we know that on the $p$-completion of diagram (3.4.1), our map (3.4.5) is, up to homotopy equivalence, the $C_{k}$ norm. On $p$-completions, however, this $C_{k}$-norm is an equivalence by Lemma (3.1).

Remark (3.5) Theorem (3.4) is also true if we use the definition of $U$ for functors with stabilization over a category, as in [8] - the same proof, with the obvious technical adjustments, works.

Remark (3.6) Commuting the order of homotopy limits, we can also write the result of Theorem (3.4) as

$$
\begin{aligned}
W(A ; M)_{p}^{\wedge} & \simeq \prod_{(k, p)=1}\left(\left(\operatorname{holim}_{\infty \leftarrow b} U^{k p^{b}}(A ; M)^{C_{p^{b}}}\right)_{p}^{\wedge}\right)^{h C_{k}} \\
& \simeq \prod_{(k, p)=1}\left(\left(\underset{\infty \leftarrow b}{\operatorname{holim}} U^{k p^{b}}(A ; M)^{C_{p^{b}}}\right)_{p}^{\wedge}\right)_{h C_{k}}
\end{aligned}
$$

\section{§4. A Filtration on $W^{(p)}(A ; M \otimes X)$}

Having expressed $W(A ; M)_{p}^{\wedge}$ in terms of $\operatorname{holim}_{\infty \leftarrow n} U^{k p^{n}}(A ; M)^{C_{p^{n}}} \simeq W^{(p)}\left(A ; M^{\hat{\otimes}_{A} k}\right)$ (as explained in Remark (3.6) above) in order to facilitate its calculation, we proceed to calculate $W^{(p)}$. We will specifically want to understand the homotopy groups of $W^{(p)}\left(A ; M \otimes S^{t}\right)$ where $p$ is a prime, and for a finite pointed simplicial set $X$. and $A$ bimodule $M, M \otimes X$ is the realization of the $M$-free simplicial set on the simplices of $X$, $n \mapsto M \wedge X_{n}$ (for any pointed simplicial set $Y$, then, the functor with stabilization $M \otimes X$ sends $Y$ to $|M(Y) \wedge X|$.$) . Observe that M \otimes X$ also inherits an $A$-bimodule structure from $M$. To understand $W^{(p)}\left(A ; M \otimes S^{t}\right)$ we will set up in this section a filtration on 
$W^{(p)}(A ; M \otimes X)$ for any FSP $A, A$-bimodule $M$, and finite pointed simplicial set $X$. The layers of this filtration are analogous to the decomposition of Theorem (2.2), which is just the discrete, zero-dimensional case $X=\{*, 1, \ldots, k\}$ for $W^{(p)}$. The filtration gives us an $E^{1}$ spectral sequence which for connected $X$ converges to $\pi_{*}\left(W^{(p)}(A ; M \otimes X)\right)$.

(4.1) The Filtration on $U^{p^{n}}$ and $W^{(p)}$

We can rewrite equation (2.2.1) as the statement that for $\underline{k}$ finite, discrete

$$
U^{n}(A ; M \otimes \underline{k}) \simeq U^{n}(A ; M) \wedge \bigwedge_{i=1}^{n} \underline{k} .
$$

This identification is natural in $\underline{k}$, and a $C_{n}$-equivalence (where $C_{n}$ acts diagonally on the right hand side). Since the functor $U^{n}(A ;-)$ commutes with realizations of simplicial sets, (4.1.1) shows that for any pointed simplicial set $X$,

$$
U^{n}(A ; M \otimes X) \simeq U^{n}(A ; M) \wedge \bigwedge_{i=1}^{n} X
$$

is a $C_{n}$-equivalence (being a homotopy direct limit, over $C_{n}$-equivariant maps, of compatible $C_{n}$-equivalences.) We will define our filtration on $U^{p^{n}}(A ; M \otimes X)$ in terms of $U^{p^{n}}(A ; M) \wedge \bigwedge_{i=1}^{p^{n}} X$. The smash product $\bigwedge_{i=1}^{p^{n}} X$ has a $C_{p^{n}}$-equivariant filtration by fixed points

$$
\left(\bigwedge_{i=1}^{p^{n}} X\right)^{C_{p^{n}}} \subset\left(\bigwedge_{i=1}^{p^{n}} X\right)^{C_{p^{n-1}}} \subset \cdots \subset\left(\bigwedge_{i=1}^{p^{n}} X\right)^{C_{p}} \subset \bigwedge_{i=1}^{p^{n}} X
$$

where the diagonal map defines natural $C_{p^{n}} / C_{p^{t}}$-equivariant homeomorphisms

$$
\bigwedge_{i=1}^{p^{t}} X \cong\left(\bigwedge_{i=1}^{p^{n}} X\right)^{C_{p^{n-t}}}
$$

and the quotients are exactly the

$$
w_{p^{t}}(X)=\frac{\left(\bigwedge_{i=1}^{p^{n}} X\right)^{C_{p^{n-t}}}}{\left(\bigwedge_{i=1}^{p^{n}} X\right)^{C_{p^{n-t-1}}}} \cong \frac{\bigwedge_{i=1}^{p^{t}} X}{\bigwedge_{i=1}^{p^{t-1}} X}
$$

of Definition (2.1).

Definition (4.2) We define a natural $C_{p^{n}}$-equivariant filtration on $U^{p^{n}}(A ; M \otimes X)$ (see (4.1.2)) by

$$
\operatorname{Fil}_{t} U^{p^{n}}(A ; M \otimes X)= \begin{cases}* & \text { if } t<0 \\ U^{p^{n}}(A ; M) \wedge\left(\bigwedge_{i=1}^{p^{n}} X\right)^{C_{p^{n-t}}} & \text { if } 0 \leq t<n \\ U^{p^{n}}(A ; M \otimes X) & \text { if } n \leq t\end{cases}
$$

The inclusions $\mathrm{Fil}_{t-1} \subseteq \mathrm{Fil}_{t}$ come from the inclusions of fixed points (4.1.3). 
Definition (4.3) We let

$$
\operatorname{Res}^{p}:\left(\operatorname{Fil}_{t} U^{p^{n}}(A ; M \otimes X)\right)^{C_{p} n} \rightarrow\left(\operatorname{Fil}_{t} U^{p^{n-1}}(A ; M \otimes X)\right)^{C_{p^{n-1}}}
$$

be the usual restriction map $\operatorname{Res}^{p}$ of Definition (1.1) if $t \geq n$, the identity map if $t<0$, and otherwise the natural composite

$$
\begin{aligned}
& \left(U^{p^{n}}(A ; M) \wedge\left(\bigwedge_{i=1}^{p^{n}} X\right)^{C_{p^{n-t}}}\right)^{C_{p^{n}}}=\left(\left(U^{p^{n}}(A ; M) \wedge\left(\bigwedge_{i=1}^{p^{n}} X\right)^{C_{p^{n-t}}}\right)^{C_{p}}\right)^{C_{p^{n}} / C_{p}} \\
& =\left(U^{p^{n}}(A ; M)^{C_{p}} \wedge\left(\bigwedge_{i=1}^{p^{n}} X\right)^{C_{p^{n-t}}}\right)^{C_{p^{n}} / C_{p}} \stackrel{\operatorname{Res} \wedge \mathrm{id}}{\longrightarrow}\left(U^{p^{n-1}}(A ; M) \wedge\left(\bigwedge_{i=1}^{p^{n}} X\right)^{C_{p^{n-t}}}\right)^{C_{p^{n}} / C_{p}} \\
& =\left(U^{p^{n-1}}(A ; M) \wedge\left(\bigwedge_{i=1}^{p^{n}} X\right)^{C_{p^{n-t}}}\right)^{C_{p^{n-1}}}
\end{aligned}
$$

where Res : $U^{p^{n}}(A ; M)^{C_{p}} \rightarrow U^{p^{n-1}}(A ; M)$ is the restriction map, analogous to those defined in Definition (1.1). Since effectively all we are doing is applying Res, we get

Lemma (4.4) For all $n, t$, and $X$, the following diagram commutes:

$$
\begin{aligned}
& \left(\operatorname{Fil}_{t} U^{p^{n}}(A ; M \otimes X)\right)^{C_{p^{n}}} \quad \hookrightarrow \quad\left(\mathrm{Fil}_{t+1} U^{p^{n}}(A ; M \otimes X)\right)^{C_{p^{n}}} \\
& \downarrow \operatorname{Res}^{p}{ }^{p} \quad \downarrow \operatorname{Res}^{p} \\
& \left(\operatorname{Fil}_{t} U^{p^{n-1}}(A ; M \otimes X)\right)^{C_{p^{n-1}}} \hookrightarrow\left(\mathrm{Fil}_{t+1} U^{p^{n-1}}(A ; M \otimes X)\right)^{C_{p^{n-1}}} .
\end{aligned}
$$

This enables us to get a filtration on $W^{(p)}$,

Definition (4.5) We have a natural filtration on $W^{(p)}(A ; M \otimes X)$ given by

$$
\operatorname{Fil}_{t} W^{(p)}(A ; M \otimes X)=\underset{\infty \leftarrow n}{\operatorname{holim}}\left(\operatorname{Fil}_{t} U^{p^{n}}(A ; M \otimes X)^{C_{p^{n}}}\right)
$$

with the inclusions $\mathrm{Fil}_{t-1} \subseteq \mathrm{Fil}_{t}$ induced by those of Definition (4.2).

Now we have two goals: The first is to check whether this filtration converges to $W^{(p)}(A ; M \otimes X)$, that is, whether

$$
\begin{aligned}
& \underset{t \rightarrow \infty}{\operatorname{hocolim}} \operatorname{Fil}_{t} W^{(p)}(A ; M \otimes X)=\underset{t \rightarrow \infty}{\operatorname{hocolim}} \operatorname{holim}_{\infty \leftarrow n}\left(\operatorname{Fil}_{t} U^{p^{n}}(A ; M \otimes X)\right)^{C_{p^{n}}} \\
& \stackrel{?}{\underset{\infty}{\operatorname{holim}} \operatorname{hocolim}}\left(\operatorname{Fil}_{t} U^{p^{n}}(A ; M \otimes X)\right)^{C_{p^{n}}} \simeq W^{(p)}(A ; M \otimes X) .
\end{aligned}
$$

This is not obvious, nor indeed always true, as we can see from the calculations we have done for $X=\underline{k}$ discrete, where the method used to obtain (2.2.2) would give us

$$
\left(\mathrm{Fil}_{t} U^{p^{n}}(A ; M \otimes X)\right)^{C_{p^{n}}} \simeq \prod_{k=0}^{t}\left(U^{p^{n}}(A ; M)^{C_{p^{n-k}}} \wedge w_{p^{k}}(X) / C_{p^{k}}\right)
$$




$$
\begin{aligned}
\underset{t \rightarrow \infty}{\operatorname{hocolim}} & \operatorname{holim}_{\infty \leftarrow n}\left(\operatorname{Fil}_{t} U^{p^{n}}(A ; M \otimes X)\right)^{C_{p^{n}}} \\
& \simeq \operatorname{hocolim}_{t \rightarrow \infty} \prod_{k=0}^{t}\left(\operatorname{holim}_{\infty \leftarrow n} U^{k p^{n}}(A ; M)^{C_{p^{n}}} \wedge w_{p^{k}}(X) / C_{p^{k}}\right) \\
& \simeq \bigvee_{k=0}^{\infty}\left(\operatorname{holim}_{\infty \leftarrow n} U^{k p^{n}}(A ; M)^{C_{p^{n}}} \wedge w_{p^{k}}(X) / C_{p^{k}}\right)
\end{aligned}
$$

whereas

$$
\begin{aligned}
& \underset{\infty \leftarrow n}{\operatorname{holim}} \operatorname{hocolim}\left(\operatorname{Fil}_{t} U^{p^{n}}(A ; M \otimes X)\right)^{C_{p^{n}}} \\
& \simeq \operatorname{holim}_{\infty \leftarrow n} \prod_{k=0}^{n}\left(U^{p^{n}}(A ; M)^{C_{p^{n-k}}} \wedge w_{p^{k}}(X) / C_{p^{k}}\right) \\
& \simeq \prod_{k=0}^{\infty}\left(\operatorname{holim}_{\infty \leftarrow n} U^{k p^{n}}(A ; M)^{C_{p^{n}}} \wedge w_{p^{k}}(X) / C_{p^{k}}\right) .
\end{aligned}
$$

(The map of (4.5.1) is the obvious inclusion of the result of (4.5.2) in that of (4.5.3).)

However, if $X$ is 0 -connected, (4.5.1) is a homotopy equivalence. We show this by pursuing our second goal: an analysis of the layers $\mathrm{Fil}_{t} W^{(p)} / \mathrm{Fil}_{t-1} W^{(p)}$, which increase in connectivity as $t$ increases when $X$ is 0 -connected.

\section{(4.6) Analyzing the Cofibers}

We start with the fundamental fibration sequence, for all $1 \leq k \leq n$, saying that for any $A$-bimodule $N$,

$$
U^{p^{n}}(A ; N)_{h C_{p^{k}}} \rightarrow U^{p^{n}}(A ; N) \stackrel{C_{p^{k}}}{\operatorname{Res}^{p}} \underset{U^{p^{n-1}}}{\longrightarrow}(A ; N)^{C_{p^{k-1}}} .
$$

This was shown in the proof of Theorem (3.2) for $k=n$, and can be shown for smaller $k$ by the same method, or by viewing $U^{p^{n}}(A ; N) \simeq U^{p^{k}}\left(A ; N^{\hat{\otimes}_{A} p^{n-k}}\right)$.

Observe that $U^{p^{n}}(A ; M \otimes X)$ commutes with colimits as a functor of $X$, so $U^{p^{n}}(A ; M \otimes$ $X)_{h C_{p^{k}}}$ does too for any $k \leq n$. We can use these two facts and (4.6.1) to show by induction that $U^{p^{n}}(A ; M \otimes X)^{C_{p^{k}}}$ commutes with colimits for any $k \leq n$.

Lemma (4.7) The functors $\left(\operatorname{Fil}_{t} U^{p^{n}}(A ; M \otimes X)\right)^{C_{p^{n}}}$ commute with colimits as functors of $X$, and we have a fibration sequence

$$
\left(\mathrm{Fil}_{t} U^{p^{n}}(A ; M \otimes X)\right)_{h C_{p^{n}}} \rightarrow\left(\operatorname{Fil}_{t} U^{p^{n}}(A ; M \otimes X)\right)^{C_{p^{n}}} \stackrel{\mathrm{Res}^{p}}{\longrightarrow}\left(\operatorname{Fil}_{t} U^{p^{n-1}}(A ; M \otimes X)\right)^{C_{p^{n-1}}},
$$

where the inclusion of the fiber is the restriction to $\mathrm{Fil}_{t}$ of the inclusion of the fiber in (4.6.1). 
Proof. By (4.1.4) and Definition (4.2), the non-trivial cofibers in the filtration on $U^{p^{n}}(A ; M \otimes X)$ are

$$
\frac{\operatorname{Fil}_{t} U^{p^{n}}(A ; M \otimes X)}{\operatorname{Fil}_{t-1} U^{p^{n}}(A ; M \otimes X)} \simeq U^{p^{n}}(A ; M) \wedge w_{p^{t}}(X), \quad 0 \leq t \leq n .
$$

Thus, we can prove that the functors $\left(\mathrm{Fil}_{t} U^{p^{n}}(A ; M \otimes X)\right)^{C_{p^{n}}}$ commute with colimits inductively, by checking that the functors $\left(U^{p^{n}}(A ; M) \wedge w_{p^{t}}(X)\right)^{C_{p^{n}}}$ commute with colimits. Now

$$
\begin{aligned}
& \left(U^{p^{n}}(A ; M) \wedge w_{p^{t}}(X)\right)^{C_{p^{n}}}=\left(\left(U^{p^{n}}(A ; M) \wedge w_{p^{t}}(X)\right)^{C_{p^{n-t}}}\right)^{C_{p^{n}} / C_{p^{n-t}}} \\
& \quad \cong\left(U^{p^{n}}(A ; M)^{C_{p^{n-t}}} \wedge w_{p^{t}}(X)\right)^{C_{p^{n}} / C_{p^{n-t}}} \simeq U^{p^{n}}(A ; M)^{C_{p^{n-t}}} \wedge_{C_{p^{t}}} w_{p^{t}}
\end{aligned}
$$

where the homeomorphism in the beginning of the second line is due to the fact that $C_{p^{n-t}}$ acts trivially on $w_{p^{t}}(X)$, and the homotopy equivalence following it is due to the fact that $C_{p^{t}} \cong C_{p^{n}} / C_{p^{n-t}}$ acts freely on $w_{p^{t}}(X)$, using

Sublemma (4.8) Let $R$ be a spectrum with a right action of a finite group $G$, and let $Y$ be a finite pointed simplicial set on which $G$ acts freely simplicially on the left. Then

$$
(R \wedge Y)^{G} \simeq R \wedge_{G} Y
$$

In terms of the spectral sequences

$$
\pi_{s}\left(R \wedge_{G} Y_{r}\right) \Rightarrow \pi_{r+s}\left(R \wedge_{G} Y\right)
$$

and

$$
\pi_{s}\left(R \wedge Y_{r}\right) \Rightarrow \pi_{r+s}(R \wedge Y)
$$

the composition

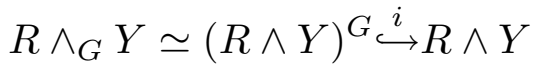

sends $[a \cdot \sigma] \mapsto \sum_{g \in G} a g^{-1} \cdot g \sigma$ for all $a \in \pi_{s} R, \sigma \in Y_{r}$.

Proof. For $Y=G_{+}$, the inclusion

$$
R \wedge G_{+}=\bigvee_{G} R \hookrightarrow \prod_{G} R
$$

is a weak equivalence which respects the $G$-action and is the spectrification map (assuming that $R$ is already an $\Omega$-spectrum). So by our definition of fixed points on a spectrum,

$$
\left(R \wedge G_{+}\right)^{G \stackrel{\text { def }}{=}}\left(\prod_{G} R\right)^{G} \cong R \cong R \wedge_{G}\left(G_{+}\right) .
$$

We can realize the homeomorphism

$$
R \wedge_{G} G_{+} \cong\left(\prod_{G} R\right)^{G}=F(G, R)^{G}
$$


(where $R$ is viewed as a left $G$-spectrum by having $h \in G$ act as $h^{-1}$ on the right) by sending $[r, g] \mapsto\left\{h g \mapsto r h^{-1}\right\}$.

Similarly, for general discrete $Y$ with free $G$-action, $Y \cong I_{+} \wedge G_{+}$(where $I$ has trivial $G$-action), and the map

$$
\begin{aligned}
R \wedge_{G} Y & \rightarrow F(Y, R)^{G} \\
{[r, i, g] } & \mapsto\left\{\begin{array}{l}
(i, h g) \mapsto r h^{-1} \\
(j, h) \mapsto * \quad \forall j \neq i
\end{array}\right\}
\end{aligned}
$$

is an equivalence $R \wedge_{G} Y \cong(R \wedge Y)^{G}$.

For higher dimensional $Y$, use the fact that the fixed points of the $G$-action on $R \wedge$ $Y$ can be computed simplicial-degree-wise (with respect to $Y$ ). The maps $R \wedge_{G} Y_{k} \rightarrow$ $F\left(Y_{k}, R\right)^{G}$ from (4.8.1) commute with the simplicial boundary maps: if $(i, g) \in Y_{k}$ and $\partial_{a}(i, g)=\left(i^{\prime}, g^{\prime}\right) \in Y_{k-1}$,

$$
\partial_{a}([r, i, g])=\left[r, i^{\prime}, g^{\prime}\right]
$$

while $\partial_{a}$ of the map sending $(i, h g)$ to $r h^{-1}$ will exactly send $\left(i^{\prime}, h g^{\prime}\right)$ to $r h^{-1}$ for all $h \in G$ and $\left(j^{\prime}, h\right)$ for $j^{\prime} \neq i^{\prime}$. Since they are homotopy equivalences on each level, they induce a homotopy equivalence on the homotopy direct limits, which are the realizations.

The result on the $E^{2}$-terms of the spectral sequences follows directly.

Since $w_{p^{k}}(X) / C_{p^{k}}$ clearly commutes with colimits, by (4.7.2) so does $\left(U^{p^{n}}(A ; M) \wedge\right.$ $\left.w_{p^{k}}(X)\right)^{C_{p^{n}}}$ and by our induction so does $\left(\mathrm{Fil}_{t} U^{p^{n}}(A ; M \otimes X)\right)^{C_{p^{n}}}$.

To prove that the sequence of maps in the statement of Lemma (4.7) is a fibration or, equivalently, a cofibration, it suffices again to check that it gives a cofibration on the layers and use induction. We look at

$\left(U^{p^{n}}(A ; M) \wedge w_{p^{t}}(X)\right)_{h C_{p^{n}}} \rightarrow\left(U^{p^{n}}(A ; M) \wedge w_{p^{t}}(X)\right)^{C_{p^{n}}} \rightarrow\left(U^{p^{n-1}}(A ; M) \wedge w_{p^{t}}(X)\right)^{C_{p^{n-1}}}$.

The restriction of $\operatorname{Res}^{p}$ to $\left(\operatorname{Fil}_{t} U^{p^{n}}(A ; M)\right)^{C_{p^{n}}}$ gives $\operatorname{Res}^{p}$ in the $U^{p^{n}}(A ; M)$ coordinate and the homeomorphism induced by $\left(\wedge_{i=1}^{p^{n}} X\right)^{C_{p^{n-t}}} \cong\left(\wedge_{i=1}^{p^{n-1}} X\right)^{C_{p^{n-1-t}}}$ in the second coordinate. Using the identification (4.7.2), we can rewrite (4.8.2)

$$
\begin{aligned}
U^{p^{n}}(A ; M)_{h C_{p^{n-t}}} \wedge_{C_{p^{t}}} w_{p^{t}}(X) & \stackrel{f}{\longrightarrow} U^{p^{n}}(A ; M)^{C_{p^{n-t}}} \wedge_{C_{p^{t}}} w_{p^{t}}(X) \\
& \stackrel{\operatorname{Res}^{p} \wedge \text { id }}{\longrightarrow} U^{p^{n-1}}(A ; M)^{C_{p^{n-1-t}}} \wedge_{C_{p^{t}}} w_{p^{t}}(X)
\end{aligned}
$$

where $f$ is the inclusion of the fiber from (4.6.1) (for $N=M$ ) smashed with the identity of $w_{p^{t}}(X) / C_{p^{t}}$. But then we just have the cofibration sequence (4.6.1) smashed with the identity of $w_{p^{t}}(X) / C_{p^{t}}$ - and so, a cofibration sequence. 
Proposition (4.9) (i) We have

$$
\operatorname{Fil}_{t} W^{(p)}(A ; M \otimes X) \simeq\left(\operatorname{holim}_{\infty \leftarrow n} U^{p^{n+t}}(A ; M)^{C_{p^{n}}} \wedge \bigwedge_{i=1}^{p^{t}} X\right)^{C_{p^{t}}},
$$

with $C_{p^{t}}$ acting on holim ${ }_{\infty \leftarrow n} U^{p^{n+t}}(A ; M)^{C_{p^{n}}} \wedge \bigwedge_{i=1}^{p^{t}} X$ diagonally.

(ii) The inclusion maps of subsequent layers of the filtration are given, using this identifcation, as

$$
\begin{aligned}
\left(\operatorname{holim}_{\infty \leftarrow n} U^{p^{n+t}}(A ; M)^{C_{p^{n}}} \wedge \bigwedge_{i=1}^{p^{t}} X\right)^{C_{p^{t}}} \simeq & \left(\left(\operatorname{holim}_{\infty \leftarrow n} U^{p^{n+t+1}}(A ; M)^{C_{p^{n}}}\right)^{C_{p}} \wedge\left(\bigwedge_{i=1}^{p^{t+1}} X\right)^{C_{p}}\right)^{C_{p} t} \\
& \left.\hookrightarrow \operatorname{holim}_{\infty \leftarrow n} U^{p^{n+t+1}}(A ; M)^{C_{p^{n}}} \wedge \bigwedge_{i=1}^{p^{t+1}} X\right)^{C_{p^{t+1}}} .
\end{aligned}
$$

(iii) The quotients of subsequent layers of the filtration are given by

$$
\frac{\operatorname{Fil}_{t} W^{(p)}(A ; M \otimes X)}{\operatorname{Fil}_{t-1} W^{(p)}(A ; M \otimes X)} \simeq \operatorname{holim}_{\infty \leftarrow n} U^{p^{n+t}}(A ; M)^{C_{p^{n}}} \wedge_{C_{p^{t}}} w_{p^{t}}(X) .
$$

Proof. We have, using Definitions (4.2), (4.3), and (4.5)

$$
\begin{aligned}
& \operatorname{Fil}_{t} W^{(p)}(A ; M \otimes X)=\operatorname{holim}_{\infty \leftarrow n}\left(\operatorname{Fil}_{t} U^{p^{n}}(A ; M \otimes X)\right)^{C_{p^{n}}} \\
& \simeq \operatorname{holim}_{\infty \leftarrow n}\left(U^{p^{n}}(A ; M) \wedge\left(\bigwedge_{i=1}^{p^{n}} X\right)^{C_{p^{n-t}}}\right)^{C_{p^{n}}} \\
& \simeq \operatorname{holim}_{\infty \leftarrow n}\left(\left(U^{p^{n}}(A ; M) \wedge\left(\bigwedge_{i=1}^{p^{n}} X\right)^{C_{p^{n-t}}}\right)^{C_{p^{n-t}}}\right)^{C_{p^{t}}} \\
& \simeq \operatorname{holim}_{\infty \leftarrow n}\left(U^{p^{n}}(A ; M)^{C_{p^{n-t}}} \wedge\left(\bigwedge_{i=1}^{p^{n}} X\right)^{C_{p^{n-t}}}\right)^{C_{p^{t}}} \\
& \simeq \operatorname{holim}_{\infty \leftarrow n}\left(U^{p^{n}}(A ; M)^{C_{p^{n-t}}} \wedge \bigwedge_{i=1}^{p^{t}} X\right)^{C_{p^{t}}} \\
&\left.\simeq \operatorname{holim}_{\infty \leftarrow n} U^{p^{n}}(A ; M)^{C_{p^{n-t}}} \wedge \bigwedge_{i=1}^{p^{t}} X\right)^{C_{p^{t}}}
\end{aligned}
$$

proving (i).

For any $n$, the inclusion

$$
\left(\mathrm{Fil}_{t} U^{p^{n}}(A ; M \otimes X)\right)^{C_{p^{n}}} \subseteq\left(\mathrm{Fil}_{t+1} U^{p^{n}}(A ; M \otimes X)\right)^{C_{p^{n}}}
$$




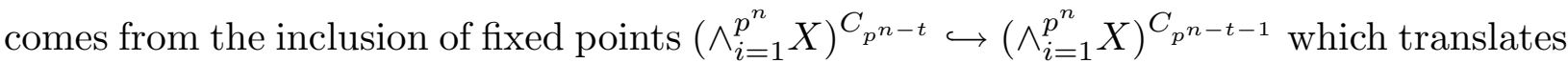
to the inclusion of $C_{p}$-fixed points $\wedge_{i=1}^{p^{t}} X \hookrightarrow \wedge_{i=1}^{p^{t+1}} X$. In terms of (4.9.1), we get the homotopy inverse limit of maps

$$
\left(U^{p^{n}}(A ; M)^{C_{p^{n-t}}} \wedge \bigwedge_{i=1}^{p^{t}} X\right)^{C_{p^{t}}} \hookrightarrow\left(\left(U^{p^{n}}(A ; M)^{C_{p^{n-t-1}}} \wedge \bigwedge_{i=1}^{p^{t+1}} X\right)^{C_{p}}\right)^{C_{p^{t}}}
$$

induced by the inclusions of $C_{p}$-fixed points in both coordinates, which is exactly (ii).

To see (iii), since homotopy inverse limits commute with finite colimits, we can take the homotopy inverse limit of the $C_{p^{n}}$-fixed points of formula (4.7.1), which we know by formula (4.7.2), and get holim $\infty_{\infty \leftarrow n} U^{p^{n+t}}(A ; M)^{C_{p^{n}}} \wedge_{C_{p^{t}}} w_{p^{t}}(X)$.

Corollary (4.10) If $X$ is n-connected, the inclusion map

$$
\mathrm{Fil}_{t} W^{(p)}(A ; M \otimes X) \rightarrow W^{(p)}(A ; M \otimes X)
$$

is $p^{t}(n+1)$-connected. In particular, if $X$ is at least 0 -connected,

$$
\underset{t \rightarrow \infty}{\operatorname{hocolim}} \operatorname{Fil}_{t} W^{(p)}(A ; M \otimes X) \stackrel{\simeq}{\longrightarrow} W^{(p)}(A ; M \otimes X) .
$$

Proof. This follows from the analysis of the cofibers in Proposition (4.9)(iii): we will show that holim $\operatorname{c\leftarrow n} U^{p^{n+s}}(A ; M)^{C_{p^{n}}} \wedge_{C_{p^{s}}} w_{p^{s}}(X)$ is $p^{s-1}(n+1)$-connected for all $s$, and apply it to all the quotients $\mathrm{Fil}_{s+1} / \mathrm{Fil}_{s}$ where $s \leq t$.

We are assuming that holim $\operatorname{\infty \leftarrow n} U^{p^{n+s}}(A ; M)^{C_{p^{n}}}$ is $(-1)$-connected. Since $\tilde{H}_{*}(X)=$ 0 for $* \leq n, \tilde{H}_{*}\left(X^{\wedge p^{s}}\right)=0$ for $* \leq p^{s}(n+1)-1$ and $\tilde{H}_{*}\left(X^{\wedge p^{s-1}}\right)=0$ for $* \leq p^{s-1}(n+1)-1$ so $\tilde{H}_{*}\left(w_{p^{s}}(X)\right)=H_{*}\left(X^{\wedge p^{s}}, X^{\wedge p^{s-1}}\right)$ is zero for $* \leq p^{s-1}(n+1)$. We have a spectral sequence

$$
\tilde{H}_{*}\left(w_{p^{s}}(X) ; \pi_{*}\left(\operatorname{holim}_{\infty \leftarrow n} U^{p^{n+s}}(A ; M)^{C_{p^{n}}}\right)\right) \Rightarrow \pi_{*}\left(\operatorname{holim}_{\infty \leftarrow n} U^{p^{n+s}}(A ; M)^{C_{p^{n}}} \wedge w_{p^{s}}(X)\right) .
$$

By the universal coefficient theorem, the $E^{2}$ term vanishes below total dimension $p^{s-1}(n+$ 1) + 1; therefore $\pi_{*}\left(\operatorname{holim}_{\infty \leftarrow n} U^{p^{n+s}}(A ; M)^{C_{p^{n}}} \wedge w_{p^{s}}(X)\right)$ does too. Since $C_{p^{s}}$ acts freely (in the based sense) on $w_{p^{s}}(X)$ and therefore also on $\operatorname{holim}_{\infty \leftarrow n} U^{p^{n+s}}(A ; M)^{C_{p^{n}}} \wedge w_{p^{s}}(X)$, the quotient by the action is the same as the homotopy quotient, and we get a spectral sequence

$$
H_{*}\left(C_{p^{s}} ; \pi_{*}\left(\operatorname{holim}_{\infty \leftarrow n} U^{p^{n+s}}(A ; M)^{C_{p^{n}}} \wedge w_{p^{s}}(X)\right)\right) \Rightarrow \pi_{*}\left(\operatorname{holim}_{\infty \leftarrow n} U^{p^{n+s}}(A ; M)^{C_{p^{n}}} \wedge_{C_{p^{s}}} w_{p^{s}}(X)\right)
$$

which again vanishes below total dimension $p^{s-1}(n+1)+1$. Therefore the spectrum $\operatorname{holim}_{\infty \leftarrow n} U^{p^{n+s}}(A ; M)^{C_{p^{n}}} \wedge_{C_{p^{s}}} w_{p^{s}}(X)$ is $p^{s-1}(n+1)$-connected. 
Corollary (4.11) If $A$ is an FSP, $M$ an A-bimodule, and $X$ a connected, finite, pointed simplicial set, we have a spectral sequence

$$
E_{r, *}^{1}=\pi_{*-r}\left(\operatorname{holim}_{\infty \leftarrow n} U^{p^{n+r}}(A ; M)^{C_{p^{n}}} \wedge_{C_{p^{r}}} w_{p^{r}}(X)\right) \Rightarrow \pi_{*} W^{(p)}(A ; M \otimes X) .
$$

\section{$\S 5$. A Calculation of $W^{(p)}\left(A ; M \otimes S^{a}\right)$}

In this section, we will use the spectral sequence from Corollary (4.11) above to calculate $W^{(p)}\left(A ; M \otimes S^{a}\right)$. We start with two lemmas which describe the $E^{1}$-term of the spectral sequence:

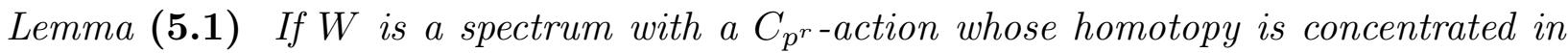
dimension 0 ,

$$
\pi_{a p^{r}}\left(W \wedge_{C_{p^{r}}} w_{p^{r}}\left(S^{a}\right)\right) \cong \operatorname{ker}\left(\pi_{0}(W) \stackrel{1-T}{\longrightarrow} \pi_{0}(W)\right),
$$

where $T=(-1)^{a\left(p^{r}-1\right)} \tau_{*}$ and $\tau: W \rightarrow W$ is the action of the generator of $C_{p^{r}}$, acting on $W$ on the right.

Proof. The filtration by skeleta gives us a complex (the Atiyah-Hirzebruch spectral sequence which vanishes off the zero'th row)

$$
\left.\left.\pi_{0}\left(W \wedge_{C_{p^{r}}}\left(\left(\widetilde{w_{p^{r}}\left(S^{a}\right.}\right)\right)_{k}\right)_{+}\right) \cong \pi_{0}(W) \otimes_{\mathbb{Z}\left[C_{p^{r}}\right]} \mathbb{Z}\left[\left(\widetilde{w_{p^{r}}\left(S^{a}\right.}\right)\right)_{k}\right]
$$

whose homology is $\pi_{*}\left(W \wedge_{C_{p^{r}}} w_{p^{r}}\left(S^{a}\right)\right)$. Here $\left.\left(\widetilde{w_{p^{r}}\left(S^{a}\right.}\right)\right)_{k}$ is the set of all non-degenerate $k$-simplices in $\left.w_{p^{r}}\left(S^{a}\right)\right)$.

We use the standard simplicial model of $S^{1}$ with one 0-simplex and one non-degenerate 1-simplex to give the simplicial structure we will use on $S^{a p^{r}} \cong\left(S^{1}\right)^{\wedge a p^{r}}$ and its quotient $w_{p^{r}}\left(S^{a}\right)=S^{a p^{r}} / S^{a p^{r-1}}$. In this model, the non-degenerate $a p^{r}$-simplices of $w_{p^{r}}\left(S^{a}\right)$ are in one-to-one correspondence with the permutation group $\Sigma_{a p^{r}}$ : to each $\sigma \in \Sigma_{a p^{r}}$ there corresponds a simplex $\rho_{\sigma}$ whose $i$ th component is the degenerate $a p^{r}$ simplex on $S^{1}$ consisting of $\sigma(i)$ zeros and $a p^{r}+1-\sigma(i)$ ones. Note that the only face of one of these simplices which is equal to $\partial_{\sigma(i)} \rho_{\sigma}$ is $\partial_{\sigma(i)} \rho_{(\sigma(i) \sigma(i)-1) \sigma}$, where $(\sigma(i) \sigma(i)-1)$ is the transposition exchanging those two numbers. (Because of this, $\sum_{\sigma \in \Sigma_{a p^{r}}}(-1)^{\sigma} \rho_{\sigma}$ generates $H_{a p^{r}}\left(S^{a p^{r}} ; \mathbb{Z}\right) \cong \mathbb{Z}$.)

Now $w_{p^{r}}\left(S^{a}\right)$, being $a p^{r}$-dimensional, has no non-degenerate $\left(a p^{r}+1\right)$-simplices. So if we want to calculate $\pi_{a p^{r}}\left(W \wedge_{C_{p^{r}}} w_{p^{r}}\left(S^{a}\right)\right)$, we only need to find the kernel of the boundary map

$$
\left.\left.\pi_{0}(W) \otimes_{\mathbb{Z}\left[C_{p^{r}}\right]} \mathbb{Z}\left[\left(\widetilde{w_{p^{r}}\left(S^{a}\right.}\right)\right)_{a p^{r}}\right] \stackrel{d}{\rightarrow} \pi_{0}(W) \otimes_{\mathbb{Z}\left[C_{p^{r}}\right]} \mathbb{Z}\left[\left(\widetilde{w_{p^{r}}\left(S^{a}\right.}\right)\right)_{a p^{r}-1}\right]
$$

The source of this map can be written as the free $\pi_{0}(W)$-module on representatives of the cosets of $C_{p^{r}}$ in $\Sigma_{a p^{r}}$. We will take as representatives all the permutations $\sigma$ for which $1 \leq \sigma(1) \leq a$. Say $\sum_{\{\sigma: 1 \leq \sigma(1) \leq a\}} \omega_{\sigma} \rho_{\sigma} \in \operatorname{ker} d$ for some choice of $\omega_{\sigma} \in \pi_{0}(W)$. We will show that we must have $\omega_{\sigma}=(-1)^{\sigma} \omega_{\text {id }}$ for all $\sigma$ with $1 \leq \sigma(1) \leq a$, and also that $T\left(\omega_{\mathrm{id}}\right)=\omega_{\mathrm{id}}$. 
The only other element in $\left.\pi_{0}(W) \otimes \mathbb{Z}\left[\left(\widetilde{w_{p^{r}}\left(S^{a}\right.}\right)\right)_{a p^{r}-1}\right]$ which can cancel $\omega_{\sigma} \partial_{\sigma(i)} \rho_{\sigma}$ is $\omega_{\sigma} \partial_{\sigma(i)} \rho_{(\sigma(i) \sigma(i)-1) \sigma}$. So after we divide out by the $C_{p^{r}}$ action, we see that $\omega_{\sigma} \partial_{\sigma(i)} \rho_{\sigma}$ can only be cancelled by the terms $-\omega_{\sigma} \tau^{-j} \partial_{\sigma(i)} \rho_{\tau^{j} \sigma}$ and $-\omega_{\sigma} \tau^{-j} \partial_{\sigma(i)} \rho_{\tau^{j}}(\sigma(i) \sigma(i)-1) \sigma$ (where we embed $C_{p^{r}} \hookrightarrow \Sigma_{a p^{r}}$ in the usual way, cycling the $p^{r} a$-tuples of coordinates). But among our representatives of the cosets of $C_{p^{r}}$ in $\Sigma_{a p^{r}}$, we have no $\rho_{\tau^{j} \sigma}$ for $j \neq 0$ (and the term corresponding to $j=0$ is the one we are trying to cancel), so we need to look for cancelling terms of the second kind.

There are two cases to consider here. If $(\sigma(i) \sigma(i)-1) \sigma$ also sends 1 to the interval $\{1,2, \ldots, a\}$, then the only member of the equivalence class of $-\omega_{\sigma} \partial_{\sigma(i)} \rho_{(\sigma(i) \sigma(i)-1) \sigma}$ which could appear in the boundary of $\sum_{\{\sigma: 1 \leq \sigma(1) \leq a\}} \omega_{\sigma} \rho_{\sigma}$ is $-\omega_{\sigma} \partial_{\sigma(i)} \rho_{(\sigma(i) \sigma(i)-1) \sigma}$ itself, which would appear as $\omega_{(\sigma(i) \sigma(i)-1) \sigma} \partial_{\sigma(i)} \rho_{(\sigma(i) \sigma(i)-1) \sigma}$. From this we deduce that $-\omega_{\sigma}=\omega_{(\sigma(i) \sigma(i)-1) \sigma}$. Since every permutation $\sigma$ with $1 \leq \sigma(1) \leq a$ can be obtained from the identity by transpositions which keep $\sigma(1)$ in $\{1,2, \ldots, a\}$, we get that a necessary condition for $\sum_{\{\sigma: 1 \leq \sigma(1) \leq a\}} \omega_{\sigma} \rho_{\sigma}$ to be in ker $d$ is that $\omega_{\sigma}=(-1)^{\sigma} \omega_{\text {id }}$ for all these $\sigma$, that is: the element of the kernel has to be of the form $\sum_{\{\sigma: 1 \leq \sigma(1) \leq a\}}(-1)^{\sigma} \omega_{\mathrm{id}} \rho_{\sigma}$.

The second case to consider is if $(\sigma(i) \sigma(i)-1) \sigma$ does not send 1 to the interval $\{1,2, \ldots, a\}$. Say for example that $\sigma(1)=a$ and $\sigma(i)=a+1$. Then the only member of the equivalence class of $-\omega_{\sigma} \partial_{\sigma(i)} \rho_{(\sigma(i) \sigma(i)-1) \sigma}$ which could appear in the boundary of $\sum_{\{\sigma: 1 \leq \sigma(1) \leq a\}} \omega_{\sigma} \rho_{\sigma}$ is $-\omega_{\sigma} \tau \partial_{\sigma(i)} \rho_{\tau^{-1}(\sigma(i) \sigma(i)-1) \sigma}$ which would appear as $\omega_{\tau^{-1}(\sigma(i) \sigma(i)-1) \sigma} \partial_{a+1} \rho_{\tau^{-1}(\sigma(i) \sigma(i)-1) \sigma}$. So for the terms to cancel, we need to have

$$
\omega_{\tau^{-1}(\sigma(i) \sigma(i)-1) \sigma}=-\omega_{\sigma} \tau^{-1}
$$

We already know that

$$
\omega_{\tau^{-1}(\sigma(i) \sigma(i)-1) \sigma}=-(-1)^{\tau}(-1)^{\sigma} \omega_{\mathrm{id}},-\omega_{\sigma} \tau^{-1}=-(-1)^{\sigma} \omega_{\mathrm{id}} \tau
$$

so we get

$$
(-1)^{\tau} \omega_{\mathrm{id}}=\omega_{\mathrm{id}} \tau,
$$

and since $\tau$ cycles $p^{r}$ a-tuples, $(-1)^{\tau}=(-1)^{a\left(p^{r}-1\right)}$, we get a second necessary condition: For $\sum_{\{\sigma: 1 \leq \sigma(1) \leq a\}}(-1)^{\sigma} \omega_{\mathrm{id}} \rho_{\sigma}$ to be in the kernel of $d$, we must have $T\left(\omega_{\mathrm{id}}\right)=\omega_{\mathrm{id}}$.

Taken together, the two necessary conditions above are sufficient: that is, for any $\omega \in \pi_{0}(W)$ with $T(\omega)=\omega$, or in other words for any $\omega \in \operatorname{ker}(1-T)$,

$$
\sum_{\{\sigma: 1 \leq \sigma(1) \leq a\}}(-1)^{\sigma} \omega \rho_{\sigma} \in \operatorname{ker} d
$$

by direct calculation, based on the above cases. If $1 \leq \sigma(1) \leq a$ but the same does not hold for the permutation $(\sigma(i) \sigma(i)-1) \sigma, \sigma(1)$ must be either $a$ or 1 , and then $\tau^{-1}(\sigma(i) \sigma(i)-1) \sigma$ or $\tau(\sigma(i) \sigma(i)-1) \sigma$, respectively, send 1 to the interval $\{1,2, \ldots, a\}$. 


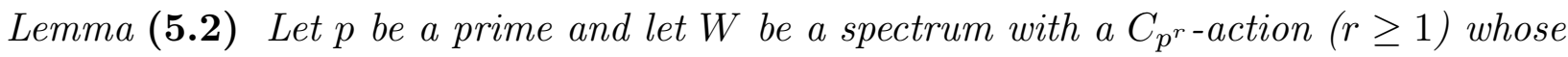
homotopy is concentrated in dimension 0 . Let $\tau: W \rightarrow W$ be the action of the generator of $C_{p^{r}}$, acting on $W$ on the right; this makes $T=(-1)^{a\left(p^{r-1}-1\right)} \tau_{*}$ act on $\pi_{0}(W)$. Then

$$
\pi_{*}\left(W \wedge_{C_{p^{r}}} w_{p^{r}}\left(S^{a}\right)\right) \cong \begin{cases}H_{*-a p^{r-1}-1}\left(C_{p^{r}} ; \pi_{0}(W)\right) & a p^{r-1}+1 \leq *<a p^{r} \\ \operatorname{ker}\left(\pi_{0}(W) \stackrel{1-(-1)^{a p^{r}-1} \tau_{*}}{\longrightarrow} \pi_{0}(W)\right) & *=a p^{r} \\ 0 & \text { otherwise. }\end{cases}
$$

Proof. The action of $C_{p^{r}}$ on $W \wedge w_{p^{r}}\left(S^{a}\right)$ is free in the pointed sense (because it is free in the pointed sense on $\left.w_{p^{r}}\left(S^{a}\right)\right)$ so the homotopy quotient is homotopy equivalent to the quotient $W \wedge_{C_{p^{r}}} w_{p^{r}}\left(S^{a}\right)$. We get a spectral sequence

$$
H_{*}\left(C_{p^{r}} ; \pi_{*}\left(W \wedge w_{p^{r}}\left(S^{a}\right)\right)\right) \Rightarrow \pi_{*}\left(W \wedge_{C_{p^{r}}} w_{p^{r}}\left(S^{a}\right)\right)
$$

Since $w_{p^{r}}\left(S^{a}\right)=S^{a p^{r}} / S^{a p^{r-1}}$ where $S^{a p^{r-1}}$ sits as the diagonal $\left(a p^{r-1}\right)$-sphere,

$$
\tilde{H}_{*}\left(w_{p^{r}}\left(S^{a}\right)\right) \cong \begin{cases}\mathbb{Z} & *=a p^{r-1}+1, a p^{r} \\ 0 & \text { otherwise }\end{cases}
$$

so

$$
\pi_{*}\left(W \wedge w_{p^{r}}\left(S^{a}\right)\right) \cong \tilde{H}_{*}\left(w_{p^{r}}\left(S^{a}\right) ; \pi_{0}(W)\right) \cong \begin{cases}\pi_{0}(W) & *=a p^{r-1}+1, a p^{r} \\ 0 & \text { otherwise }\end{cases}
$$

We need to know how the generator $\tau$ of $C_{p^{r}}$ acts on (5.2.2). In the proof of the previous lemma, it was mentioned that $H_{a p^{r}}\left(S^{a p^{r}} ; \mathbb{Z}\right) \cong H_{a p^{r}}\left(w_{p^{r}}\left(S^{a}\right) ; \mathbb{Z}\right)$ is generated by the class of the cycle $\sum_{\sigma \in \Sigma_{a p^{r}}}(-1)^{\sigma} \rho_{\sigma}$, and $\tau$ acts on this by sending $\rho_{\sigma} \mapsto \rho_{\tau \sigma}$ where $\tau$ cycles the $p^{r}$ $a$-tuples of coordinates. Since the sign of this cyclic permutation is $(-1)^{\left(p^{r}-1\right) a}$, we get that the generator of the $C_{p^{r}}$ acts as $(-1)^{\left(p^{r}-1\right) a} \tau_{*}$ on the copy of $\pi_{0}(W)$ in $\pi_{a p^{r}}\left(W \wedge w_{a p^{r}}\left(S^{a}\right)\right)$.

By naturality of the boundary map in the long exact homology sequence of a couple, the action of $C_{p^{r}}$ on $\pi_{a p^{r-1}+1}\left(W \wedge w_{a p^{r}}\left(S^{a}\right)\right) \cong H_{a p^{r-1}+1}\left(S^{a p^{r}} / S^{a p^{r-1}} ; \pi_{0}(W)\right)$ agrees with

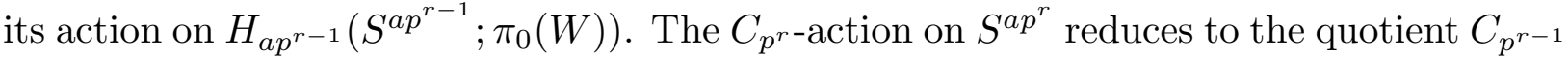
action on $S^{a p^{r-1}} \cong\left(S^{a p^{r}}\right)^{C_{p}}$, which acts as $(-1)^{a\left(p^{r-1}-1\right)} \tau_{*}$ (the parity distinction between $p^{r}$ and $p^{r-1}$ is of course unnecessary unless $p$ is even, $a$ is odd, and $\left.r=1\right)$.

Understanding the $E^{2}$-term of this spectral sequence completes the calculation, since no differentials or extensions can affect the terms of total degree less than $a p^{r}$ sitting in $E_{j, a p^{r-1}+1}^{2} \cong E_{j, a p^{r-1}+1}^{\infty}, 0 \leq j<a p^{r}-a p^{r-1}-1$, since Lemma (5.1) gives us the correct answer in total degree $a p^{r}$, and since above degree $a p^{r}$ we do not expect any homotopy $\left(w_{p^{r}}\left(S^{a}\right)\right.$ is an $a p^{r}$-dimensional complex and the homotopy of $W$ is concentrated in dimension 0$)$. 
Theorem (5.3) Assume that $p$ is a prime, $A$ is an FSP and $M$ is an A-bimodule so that the homotopy of $W^{(p)}\left(A ; M^{\hat{\otimes}_{A} p^{r}}\right)$ is concentrated in dimension 0 for all $r \geq 0$. Set $W_{r}=$ $\pi_{0}\left(\operatorname{holim}_{\infty \leftarrow n} U^{p^{n+r}}(A ; M)^{C_{p^{n}}}\right) \cong \pi_{0}\left(W^{(p)}\left(A ; M^{\hat{\otimes}_{A} p^{r}}\right)\right)$ for all $r \geq 0$. Let $v_{p}: W_{r} \rightarrow W_{r-1}$ denote the map induced by the $p$ Verschiebung map (1.3), and let $T: W_{r} \rightarrow W_{r}$ denote the map $(-1)^{a p^{r-1}-1} \tau_{*}$, where $\tau$ : $\operatorname{holim}_{\infty \leftarrow n} U^{p^{n+r}}(A ; M)^{C_{p^{n}}} \rightarrow \operatorname{holim}_{\infty \leftarrow n} U^{p^{n+r}}(A ; M)^{C_{p^{n}}}$ generates the $C_{p^{r}}$-action. Then $T$ generates a $C_{p^{r}}$-action on $W_{r}$, and we have that

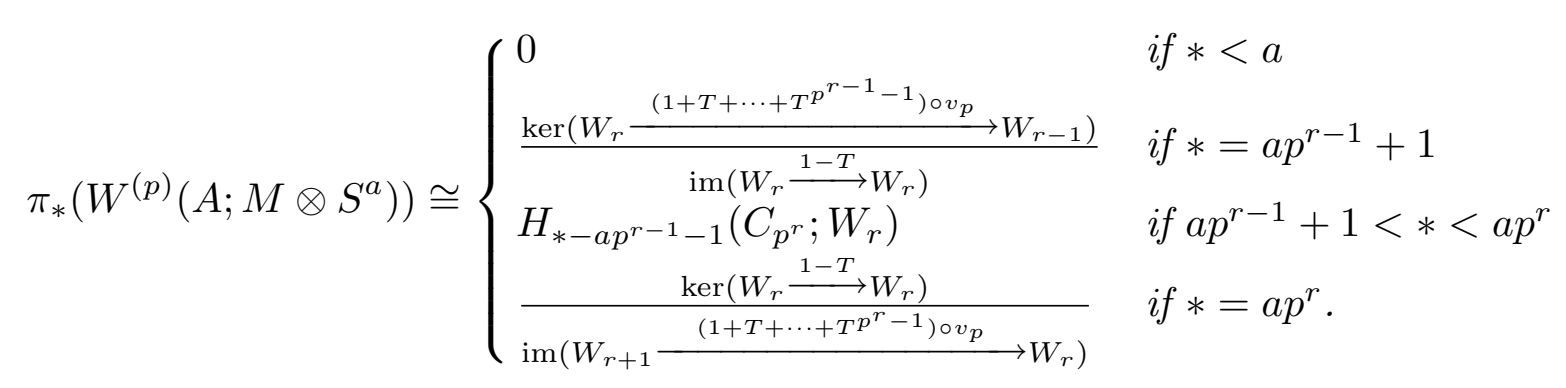

Proof. By Corollary (4.11), we have a spectral sequence with

$$
E_{r, *}^{1}=\pi_{*-r}\left(\operatorname{holim}_{\infty \leftarrow n} U^{p^{n+r}}(A ; M)^{C_{p^{n}}} \wedge_{C_{p^{r}}} w_{p^{r}}\left(S^{a}\right)\right) \Rightarrow \pi_{*} W^{(p)}\left(A ; M \otimes S^{a}\right) .
$$

By Lemma (5.2), this has the form

$$
E_{r, s}^{1} \cong \begin{cases}W_{0} & r=0, s=a \\ H_{r+s-a p^{r-1}-1}\left(C_{p^{r}} ; \pi_{0}\left(W_{r}\right)\right) & 1 \leq r, a p^{r-1}+1 \leq r+s<a p^{r} \\ \operatorname{ker}\left(W_{r} \stackrel{1-T}{\longrightarrow} W_{r}\right) & 1 \leq r, r+s=a p^{r} \\ 0 & \text { otherwise. }\end{cases}
$$

Because of the staircase form of this spectral sequence, the only possible nontrivial differentials are

$$
d_{r, a p^{r-1}+1-r}^{1}: E_{r, a p^{r-1}+1-r}^{1} \rightarrow E_{r-1, a p^{r-1}+1-r}^{1},
$$

and there are no possible extension problems.

We know that

$$
E_{r, a p^{r-1}+1-r}^{1}=H_{0}\left(C_{p^{r}} ; W_{r}\right)=W_{r} / \operatorname{im}\left(W_{r} \stackrel{1-T}{\longrightarrow} W_{r}\right)
$$

and

$$
E_{r-1, a p^{r-1}+1-r}^{1}=\operatorname{ker}\left(W_{r-1} \stackrel{1-T}{\longrightarrow} W_{r-1}\right) .
$$

Let $\alpha=\sum_{\sigma \in \Sigma_{a p^{r-1}}}(-1)^{\sigma} \rho_{\sigma}$ be a generator of the top simplicial homology class of the diagonal $a p^{r-1}$-sphere $S^{a p^{r-1}} \hookrightarrow S^{a p^{r}}$, like in the proof of Lemma (5.1). Let $\beta=\sum_{i=1}^{n} a_{i} \phi_{i}$ be a simplicial $\left(a p^{r-1}+1\right)$-chain on $S^{a p^{r}}$ with integer coefficients such that $\partial \beta=\alpha$. Let

$$
c: S^{a p^{t}} \rightarrow w_{p^{t}}\left(S^{a}\right)=S^{a p^{t}} / S^{a p^{t-1}}
$$


be the collapse maps and

$$
q: \operatorname{holim}_{\infty \leftarrow n} U^{p^{n+t}}(A ; M)^{C_{p^{n}}} \wedge w_{p^{t}}\left(S^{a}\right) \rightarrow \operatorname{holim}_{\infty} U^{p^{n+t}}(A ; M)^{C_{p^{n}}} \wedge_{C_{p^{t}}} w_{p^{t}}\left(S^{a}\right)
$$

be the quotient maps for $t=r-1, r$.

By looking at the inclusion of the first column into the spectral sequence (5.2.1) or by looking at the universal coefficients spectral sequence for $\mathbb{Z}$ over $\mathbb{Z}\left[C_{p^{r}}\right]$ (which turns out to be the same calculation), the map induced by $q$ on the lowest nontrivial homotopy group

$$
\begin{aligned}
q_{*}: \pi_{a p^{r-1}+1} & \left(\underset{\infty \leftarrow n}{\operatorname{holim}} U^{p^{n+r}}(A ; M)^{C_{p^{n}}} \wedge w_{p^{r}}\left(S^{a}\right)\right) \cong W_{r} \\
& \rightarrow \pi_{a p^{r-1}+1}\left(\operatorname{holim}_{\infty \leftarrow n} U^{p^{n+r}}(A ; M)^{C_{p^{n}}} \wedge_{C_{p^{r}}} w_{p^{t}}\left(S^{a}\right)\right) \cong W_{r} / \operatorname{im}\left(W_{r} \stackrel{1-T}{\longrightarrow} W_{r}\right)
\end{aligned}
$$

is the obvious quotient map. Filtering $w_{p^{r}}\left(S^{a}\right)$ by simplicial skeleta gives a complex

$$
\pi_{0}\left(\operatorname{holim}_{\infty \leftarrow n} U^{p^{n+r}}(A ; M)^{C_{p^{n}}} \wedge\left(w_{p^{r}}\left(S^{a}\right)\right)_{k}\right)
$$

whose homology is $\pi_{*}\left(\operatorname{holim}_{\infty \leftarrow n} U^{p^{n+r}}(A ; M)^{C_{p^{n}}} \wedge w_{p^{r}}\left(S^{a}\right)\right)$ and a complex

$$
\pi_{0}\left(\operatorname{holim}_{\infty \leftarrow n} U^{p^{n+r}}(A ; M)^{C_{p^{n}}} \wedge_{C_{p^{r}}}\left(w_{p^{r}}\left(S^{a}\right)\right)_{k}\right)
$$

whose homology is $\pi_{*}\left(\operatorname{holim}_{\infty \leftarrow n} U^{p^{n+r}}(A ; M)^{C_{p^{n}}} \wedge_{C_{p^{r}}} w_{p^{r}}\left(S^{a}\right)\right)$.

In these terms, the classes in $\pi_{a p^{r-1}+1}\left(\operatorname{holim}_{\infty \leftarrow n} U^{p^{n+r}}(A ; M)^{C_{p^{n}}} \wedge w_{p^{r}}\left(S^{a}\right)\right)$ are represented by $w \cdot c_{*} \beta$ for all $w \in W_{r}$, whose images will be $q_{*}\left(w \cdot c_{*} \beta\right)$. By the equivalence of Sublemma (4.8), $q_{*}\left(w \cdot c_{*} \beta\right) \in \pi_{0}\left(\operatorname{holim}_{\infty \leftarrow n} U^{p^{n+r}}(A ; M)^{C_{p^{n}}} \wedge_{C_{p^{r}}}\left(w_{p^{r}}\left(S^{a}\right)\right)_{a p^{r-1}+1}\right)$ corresponds to

$$
\sum_{j=0}^{p^{r}-1} w \tau^{-j} \cdot \tau^{j} c_{*} \beta \in \pi_{0}\left(\left(\operatorname{holim}_{\infty \leftarrow n} U^{p^{n+r}}(A ; M)^{C_{p^{n}}} \wedge\left(w_{p^{r}}\left(S^{a}\right)\right)_{a p^{r-1}+1}\right)^{C_{p^{r}}}\right),
$$

where we denote elements of $\pi_{0}\left(\left(\operatorname{holim}_{\infty \leftarrow n} U^{p^{n+r}}(A ; M)^{C_{p^{n}}} \wedge\left(w_{p^{r}}\left(S^{a}\right)\right)_{a p^{r-1}+1}\right)^{C_{p^{r}}}\right)$ by the names they get from the inclusion into $\operatorname{holim}_{\infty \leftarrow n} U^{p^{n+r}}(A ; M)^{C_{p^{n}}} \wedge\left(w_{p^{r}}\left(S^{a}\right)\right)_{a p^{r-1}+1}$.

Our aim is to find $d_{r, a p^{r-1}+1-r}^{1}\left(q_{*}\left(w \cdot c_{*} \beta\right)\right)$. By Proposition (4.9)(i)-(ii), the differential (5.3.2) is induced by the boundary map in the long exact sequence of the cofibration

$$
\begin{aligned}
& \left(\operatorname{holim}_{\infty \leftarrow n} U^{p^{n+r-1}}(A ; M)^{C_{p^{n}}} \wedge \bigwedge_{i=1}^{p^{r-1}} S^{a}\right)^{C_{p^{r-1}}} \\
& \simeq\left(\left(\operatorname{holim}_{\infty \leftarrow n} U^{p^{n+r}}(A ; M)^{C_{p^{n}}}\right)^{C_{p}} \wedge\left(\bigwedge_{i=1}^{p^{r}} S^{a}\right)^{C_{p}}\right)^{C_{p^{r-1}}} \\
& \hookrightarrow\left(\operatorname{holim}_{\infty \leftarrow n} U^{p^{n+r}}(A ; M)^{C_{p^{n}}} \wedge \bigwedge_{i=1}^{p^{r}} S^{a}\right)^{C_{p^{r}}} .
\end{aligned}
$$


Note that

$$
\begin{aligned}
& \left(\underset{\infty \leftarrow n}{\operatorname{holim}} U^{p^{n+r}}(A ; M)^{C_{p^{n}}} \wedge\left(\bigwedge_{i=1}^{p^{r}} S^{a}\right)^{C_{p}}\right)^{C_{p^{r}}} \\
& =\left(\left(\operatorname{holim}_{\infty \leftarrow n} U^{p^{n+r}}(A ; M)^{C_{p^{n}}} \wedge\left(\bigwedge_{i=1}^{p^{r}} S^{a}\right)^{C_{p}}\right)^{C_{p}}\right)^{C_{p^{r-1}}} \\
& =\left(\left(\operatorname{holim}_{\infty \leftarrow n} U^{p^{n+r}}(A ; M)^{C_{p^{n}}}\right)^{C_{p}} \wedge\left(\bigwedge_{i=1}^{p^{r}} S^{a}\right)^{C_{p}}\right)^{C_{p^{r-1}}},
\end{aligned}
$$

so the cofibration (5.3.5) can also be thought of as

$$
\left(\operatorname{holim}_{\infty \leftarrow n} U^{p^{n+r}}(A ; M)^{C_{p^{n}}} \wedge\left(\bigwedge_{i=1}^{p^{r}} S^{a}\right)^{C_{p}}\right)^{C_{p^{r}}} \hookrightarrow\left(\operatorname{holim}_{\infty \leftarrow n} U^{p^{n+r}}(A ; M)^{C_{p^{n}}} \wedge \bigwedge_{i=1}^{p^{r}} S^{a}\right)^{C_{p^{r}}}
$$

(leading to another proof of Theorem (4.9)(iii)).

We get that

$$
d_{r, a p^{r-1}+1-r}^{1}\left(q_{*}\left(w \cdot c_{*} \beta\right)\right)=q_{*} c_{*}\left(\partial\left(\sum_{j=0}^{p^{r}-1} w \tau^{-j} \cdot \tau^{j} \beta\right)\right)=q_{*} c_{*}\left(\sum_{j=0}^{p^{r}-1} w \tau^{-j} \cdot \tau^{j} \alpha\right) .
$$

But recall that $\alpha$ is a sum of simplices in $S^{a p^{r-1}}=\left(S^{a p^{r}}\right)^{C_{p}}$, so

$$
\sum_{j=0}^{p^{r}-1} w \tau^{-j} \cdot \tau^{j} \alpha=\sum_{j=0}^{p^{r-1}-1}\left(\sum_{k=0}^{p-1} w \tau^{k p^{r-1}}\right) \tau^{-j} \cdot \tau^{j} \alpha
$$

Now $\sum_{k=0}^{p-1} w \tau^{k p^{r-1}}$ is the $C_{p}$ norm of $w$, which lands in the $C_{p}$-fixed points of the spectrum, $\left(\operatorname{holim}_{\infty \leftarrow n} U^{p^{n+r}}(A ; M)^{C_{p^{n}}}\right)^{C_{p}} \cong \operatorname{holim}_{\infty \leftarrow n} U^{p^{n+r-1}}(A ; M)^{C_{p^{n}}}$. Recall that $\operatorname{holim}_{\infty \leftarrow n} U^{p^{n+r-1}}(A ; M)^{C_{p^{n}}}$ is in fact the coefficient spectrum in the $(r-1)$ 'st column, which we have identified into the $C_{p}$-fixed points of holim ${ }_{\infty \leftarrow n} U^{p^{n+r}}(A ; M)^{C_{p^{n}}}$ in $(5.3 .5)$ above to make it easier to understand the cofibration boundary map. If we want, then, to view $\sum_{k=0}^{p-1} w \tau^{k p^{r-1}}$ as an element of $\pi_{0}\left(\operatorname{holim}_{\infty \leftarrow n} U^{p^{n+r-1}}(A ; M)^{C_{p^{n}}}\right)$, it is exactly $v_{p}(w)$. So

$$
\sum_{j=0}^{p^{r}-1} w \tau^{-j} \cdot \tau^{j} \alpha=\sum_{j=0}^{p^{r-1}-1} v_{p}(w) \tau^{-j} \cdot \tau^{j} \alpha \in \pi_{0}\left(\underset{\infty \leftarrow n}{\operatorname{holim}} U^{p^{n+r-1}}(A ; M)^{C_{p^{n}}} \wedge\left(S^{a p^{r-1}}\right)_{a p^{r-1}}\right) .
$$

By Sublemma (4.8) again, the element

$$
c_{*}\left(\sum_{j=0}^{p^{r-1}-1} v_{p}(w) \tau^{-j} \cdot \tau^{j} \alpha\right)=\sum_{j=0}^{p^{r-1}-1} v_{p}(w) \tau^{-j} \cdot \tau^{j} c_{*} \alpha
$$


is in the image of the fixed points $\left(\operatorname{holim}_{\infty \leftarrow n} U^{p^{n+r-1}}(A ; M)^{C_{p^{n}}} \wedge\left(S^{a p^{r-1}}\right)_{a p^{r-1}}\right)^{C_{p^{r-1}}}$ and corresponds by the equivalence there to $q_{*}\left(v_{p}(w) \cdot c_{*} \alpha\right)$. So

$$
d_{r, a p^{r-1}+1-r}^{1}\left(q_{*}\left(w \cdot c_{*} \beta\right)\right)=q_{*}\left(v_{p}(w) \cdot c_{*} \alpha\right) .
$$

Recall now that

$$
\alpha=\sum_{\sigma \in \Sigma_{a p^{r}-1}}(-1)^{\sigma} \rho_{\sigma}=\sum_{\ell=0}^{p^{r-1}-1}(-1)^{a\left(p^{r-1}-1\right) \ell} \tau^{\ell}\left(\sum_{\{\sigma: 1 \leq \sigma(1) \leq a\}}(-1)^{\sigma} \rho_{\sigma}\right) .
$$

So

$$
\begin{aligned}
q_{*}\left(v_{p}(w) \cdot c_{*} \alpha\right)= & q_{*}\left(\sum_{\ell=0}^{p^{r-1}-1}(-1)^{a\left(p^{r-1}-1\right) \ell} v_{p}(w) \cdot \tau^{\ell} \sum_{\{\sigma: 1 \leq \sigma(1) \leq a\}}(-1)^{\sigma} c_{*} \rho_{\sigma}\right) \\
& =q_{*}\left(\left(\sum_{\ell=0}^{p^{r-1}-1}(-1)^{a\left(p^{r-1}-1\right) \ell} v_{p}(w) \tau^{\ell}\right) \cdot \sum_{\{\sigma: 1 \leq \sigma(1) \leq a\}}(-1)^{\sigma} c_{*} \rho_{\sigma}\right) \\
& =q_{*}\left(\left(1+T+\cdots T^{p^{r-1}-1}\right) v_{p}(w) \cdot \sum_{\{\sigma: 1 \leq \sigma(1) \leq a\}}(-1)^{\sigma} c_{*} \rho_{\sigma}\right)
\end{aligned}
$$

which, in terms of identifying $\pi_{a p^{r-1}}\left(\operatorname{holim}_{\infty \leftarrow n} U^{p^{n+r-1}}(A ; M)^{C_{p^{n}}} \wedge_{C_{p^{r-1}}} w_{p^{r-1}}\left(S^{a}\right)\right)$ as $\operatorname{ker}\left(W_{r-1} \stackrel{1-T}{\longrightarrow} W_{r-1}\right)$ in (5.3.4) by the analysis of Lemma (5.1), corresponds to $(1+T+$ $\left.\cdots T^{p^{r-1}-1}\right) v_{p}(w)$.

We have, then, shown that in the terms of (5.3.3) and (5.3.4), $d_{r, a p^{r-1}+1-r}^{1}=$ $\left(1+T+\cdots T^{p^{r-1}-1}\right) \circ v_{p}$.

Corollary (5.4) Assume that $A$ is an FSP for which the homotopy of $W^{(p)}(A ; A)$ is concentrated in dimension zero; set $W=\pi_{0}\left(W^{(p)}(A ; A)\right)$. (i) If $p$ is an odd prime, or if a is even,

$$
\pi_{*}\left(W^{(p)}\left(A ; A \otimes S^{a}\right)\right) \cong \begin{cases}0 & \text { if } *<a \\ \operatorname{ker}\left(p^{r-1} v_{p}\right) & \text { if } * a p^{r-1}+1 \\ W / p^{r} W & \text { if } a p^{r-1}<*<a p^{r}, *-a p^{r} \text { even } \\ \operatorname{Ann}_{W} p^{r} & \text { if } a p^{r-1}<*<a p^{r}, *-a p^{r} \text { odd } \\ W / p^{r} v_{p}(W) & \text { if } *=a p^{r}\end{cases}
$$

(ii) If $p=2$ and $a$ is odd,

$$
\pi_{*}\left(W^{(2)}\left(A ; A \otimes S^{a}\right)\right) \cong \begin{cases}0 & \text { if } *<a \\ W / v_{2}(W) & \text { if } *=a \\ \operatorname{ker}\left(v_{2}\right) & \text { if } *=a+1 \\ W / 2 W & \text { if } *>a \text { is odd } \\ \operatorname{Ann}_{W} 2 & \text { if } *>a+1 \text { is even }\end{cases}
$$


where $v_{p}$ is the Verschiebung map of Definition (1.3).

Proof. The crucial observation is that the action of any element of $C_{p^{r}}$ on $W^{(p)}(A ; A) \cong$ $\operatorname{holim}_{\infty \leftarrow n} U^{p^{n+r}}(A ; A)^{C_{p^{n}}}$ is homotopic to the identity for all $n$. Once this is known, $T$ from the result of (5.3) acts as $(-1)^{a\left(p^{r-1}-1\right)}$, and the answer follows (for the case of $p=2$, $a$ odd observe that if $r>1,\left(1+T+\cdots+T^{p^{r-1}-1}\right) \circ v_{2}=0$, but if $r=1$ we get $\left.1 \circ v_{2}=v_{2}\right)$.

The $C_{p^{r}}$ action we have on $\operatorname{holim}_{\infty \leftarrow n} U^{p^{n+r}}(A ; A)^{C_{p^{n}}}$ is exactly the $C_{p^{r}}$ action $W^{(p)}(A ; A)$ gets from its Connes cyclic structure. The point is that for any $n$, the $U^{p^{n+r}}(A ; A)$ is exactly the $p^{r}$ th edgewise subdivision of $U^{p^{n}}(A ; A)$ (see [1] for an analogous calculation; in fact, each $U^{p^{n}}(A ; A)$ is the $p^{n}$ th edgewise subdivision of $\operatorname{THH}(A)$, the example discussed there). This identification preserves the $C_{p^{n+r}}$ action, and thus the $C_{p^{r}}$ action on the $C_{p^{n}}$ fixedpoints; it also commutes with the restriction maps $\operatorname{Res}_{p}$ as $n$ varies. Thus we get a Connes cyclic structure on the homotopy inverse limit.

The action of any element in $C_{p^{r}}$ on the realization of a cyclic set in Connes's sense is homotopic to the identity because the $C_{p^{r}}$ action is the restriction of an $S^{1}$-action.

Corollary (5.5) Let $A$ be the FSP associated to a perfect field of characteristic $p>0$, $A(|X|)=.|A[X.] / A \cdot *|$, and let $W(A)$ denote the (algebraic) ring of $p$-typical Witt vectors of $A$. Then if $p$ is odd or a is even,

$$
\pi_{*}\left(W^{(p)}\left(A ; A \otimes S^{a}\right)\right) \cong \begin{cases}0 & \text { if } *<a \text { or } *-\text { ap odd } \\ W(A) / p^{r} W(A) & \text { if } a p^{r-1}<* \leq a p^{r}, *-\text { ap even }\end{cases}
$$

Proof. This follows from Corollary (5.4) by results of Hesselholt and Madsen, who have also proved the case $a=1$ of this Corollary. The facts we need are that the homotopy of $W^{(p)}(A ; A)$ is concentrated in dimension zero (note that $W^{(p)}(A ; A)$ is $\mathrm{TR}^{(p)}(A)$ in the notation of [1] and [6]), which is Theorem 5.5 in [6]; that $\pi_{0}\left(W^{(p)}(A ; A)\right)$ is isomorphic to the $p$-Witt vectors of $A$ in an isomorphism which carries our Verschiebung map to the algebraic one, which is the inverse limit of Theorem 3.3 in [6]; and that in the algebraic $p$-Witt vectors of $A, v_{p}$ and multiplication by $p$ are both injections and their images are the same.

From pages 48-49 of [6], we have formulas for the Verschiebung and Frobenius maps on the $p$-typical Witt vectors in terms of the Witt coordinates: For any ring $A$,

$$
v_{p}\left(a_{0}, a_{1}, a_{2}, \ldots\right)=\left(0, a_{o}, a_{1}, a_{2}, \ldots\right)
$$

and for $\mathbf{F}_{p}$-algebras,

$$
F\left(a_{0}, a_{1}, a_{2}, \ldots\right)=\left(a_{0}^{p}, a_{1}^{p}, a_{2}^{p}, \ldots\right) .
$$

It is clear from these formulas that $v_{p}$ is always injective, and that for a perfect characteristic $p$ field, $F$ is an isomorphism. Formula (10) in [6] is that the composition $F \circ v_{p}$ is multiplication by $p$. Thus multiplication by $p$ is also injective on the $p$-typical Witt vectors of a perfect characteristic $p$ field. We can write $v_{p}$ as $F^{-1}$ composed with multiplication by $p$, but since multiplication by $p$ commutes with any ring homomorphism, we can switch the order and write $v_{p}$ as multiplication by $p$ composed with the isomorphism $F^{-1}$, which shows that the images of $v_{p}$ and multiplication by $p$ coincide. 
Corollary (5.6) Let A be the FSP associated as above to a perfect field of characteristic 2. Then for a odd,

$$
\pi_{*}\left(W^{(2)}\left(A ; A \otimes S^{a}\right)\right) \cong \begin{cases}0 & \text { if } *<a \text { or } * \text { is even } \\ W(A) / 2 W(A) & \text { if } * \geq a \text { is odd }\end{cases}
$$

Proof. The $a=1$ case of this is again due to Hesselholt and Madsen, and the proof is analogous to that of Corollary (5.5).

§6. Calculations of $\left(\left(\operatorname{holim}_{\infty \leftarrow n} U^{t p^{n}}\left(A ; A \otimes S^{a}\right)^{C_{p^{n}}}\right)_{p}^{\wedge}\right)_{h C_{t}}$

In our calculation of reduced algebraic K-groups of extensions by free modules of finite rank, we will use the decomposition from Remark (3.6), so we will need to study homotopy quotients $\left(\left(\operatorname{holim}_{\infty \leftarrow n} U^{p^{n+t}}(A ; M)^{C_{p} n}\right)_{p}^{\wedge}\right)_{h C_{t}}$, for $t$ coprime to $p$. In the case $M=A$, they can be described easily, and this description will be used in our calculation for $M=A^{\oplus k}$.

Lemma (6.1) Let $t$ be coprime to $p$. If $t$ is odd, or a is even, the obvious map from a spectrum to its $C_{t}$ homotopy orbits induces an equivalence

$$
\left(U^{t p^{b}}\left(A ; A \otimes S^{a}\right)^{C_{p^{b}}}\right)_{p}^{\wedge} \stackrel{\simeq}{\longrightarrow}\left(\left(U^{t p^{b}}\left(A ; A \otimes S^{a}\right)^{C_{p^{b}}}\right)_{p}^{\wedge}\right)_{h C_{t}}
$$

for any $b \geq 0$. Taking the homotopy inverse limit of these equivalences, we get an equivalence

$$
\begin{aligned}
W^{(p)}\left(A ; A \otimes S^{a t}\right)_{p}^{\wedge} & \simeq\left(\operatorname{holim}_{\infty \leftarrow n} U^{p^{n+t}}\left(A ; A \otimes S^{a}\right)^{C_{p^{n}}}\right)_{p}^{\wedge} \\
& \simeq\left(\left(\operatorname{holim}_{\infty \leftarrow n} U^{p^{n+t}}\left(A ; A \otimes S^{a}\right)^{C_{p^{n}}}\right)_{p}^{\wedge}\right)_{h C_{t}} .
\end{aligned}
$$

Proof. We have the usual fibrations (which, for spectra, agree with cofibrations)

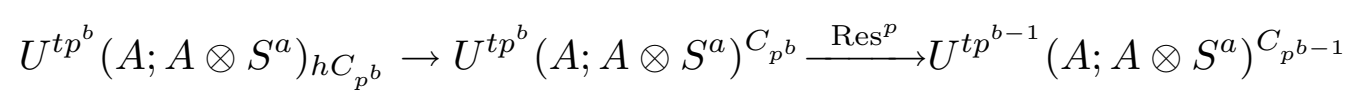

using the method described in the proof of Theorem (3.2). This means that $\left(\left(U^{t p^{b}}(A ; A \otimes\right.\right.$ $\left.\left.\left.S^{a}\right)^{C_{p^{b}}}\right)_{p}^{\wedge}\right)_{h C_{t}}$ is built out of pieces of the form $\left(\left(U^{t p^{n}}\left(A ; A \otimes S^{a}\right)_{h C_{p^{n}}}\right)_{p}^{\wedge}\right)_{h C_{t}}$ for $n \leq b$. We will show that the usual map from a space to its homotopy quotient

$$
\left(U^{t p^{n}}\left(A ; A \otimes S^{a}\right)_{h C_{p^{n}}}\right)_{p}^{\wedge} \rightarrow\left(\left(U^{t p^{n}}\left(A ; A \otimes S^{a}\right)_{h C_{p^{n}}}\right)_{p}^{\wedge}\right)_{h C_{t}}
$$

is an equivalence for any $n$.

Since $t$ is coprime to $p$, the spectral sequence

$$
H_{*}\left(C_{t} ; \pi_{*}\left(\left(U^{t p^{n}}\left(A ; A \otimes S^{a}\right)_{h C_{p^{n}}}\right)_{p}^{\wedge}\right)\right) \Rightarrow \pi_{*}\left(\left(\left(U^{t p^{n}}\left(A ; A \otimes S^{a}\right)_{h C_{p^{n}}}\right)_{p}^{\wedge}\right)_{h C_{t}}\right)
$$


is non-zero only in the zero'th column, where consists of

$$
\pi_{*}\left(\left(U^{t p^{n}}\left(A ; A \otimes S^{a}\right)_{h C_{p^{n}}}\right)_{p}^{\wedge}\right)_{C_{t}},
$$

and is the image of $\pi_{*}\left(\left(U^{t p^{n}}\left(A ; A \otimes S^{a}\right)_{h C_{p^{n}}}\right)_{p}^{\wedge}\right)$ under the map (6.1.1). We will show that the action of $C_{t}$ is homotopic to the identity on $\pi_{*}\left(\left(U^{t p^{n}}\left(A ; A \otimes S^{a}\right)_{h C_{p^{n}}}\right)_{p}^{\wedge}\right)$ :

Recall that by (4.1.2), there is a $C_{t p^{n}}$-equivalence

$$
U^{t p^{n}}\left(A ; A \otimes S^{a}\right) \simeq U^{t p^{n}}(A ; A) \wedge \bigwedge_{i=1}^{t p^{n}} S^{a}
$$

where $C_{t p^{n}}$ acts diagonally on the right hand side. Note that $U^{t p^{n}}(A ; A)$ is just the $\left(t p^{n}\right)^{\prime}$ th edgewise subdivision of $\operatorname{THH}(A)$ from [1]; the $C_{t}$-action on it is the restriction of Connes' $S^{1}$-action on a cyclic object. Therefore the action of the generator of $C_{t}$ is homotopic to the identity via the action of the elements on the arc in $S^{1}$ from 1 to $e^{\frac{2 \pi i}{t}}$. Note that if we use a homotopy of this kind, it is $C_{p^{n}}$-equivariant (where $C_{p^{n}}$ also acts as a subgroup of $S^{1}$ ) because $S^{1}$ is abelian.

The generator of $C_{t}$ acts on $\bigwedge_{i=1}^{t p^{n}} S^{a}$ by cycling $t$ blocks of $p^{n} a$ coordinates. We can arrange the coordinates as $\bigwedge_{i=1}^{p^{n}} S^{\text {at }}$ where the $C_{t}$ action cycles the $t$ blocks of $a$ coordinates in each of the $p^{n}$ components. So on each $S^{a t}$, a generator acts by a permutation whose sign is $(t-1) a$ which is even since $t$ is odd or $a$ even. This means that on each $S^{a t}$ there is a homotopy from the action of a generator of $C_{t}$ to the identity. Applying this homotopy simultaneously on all the $p^{n}$ coordinates in $\bigwedge_{i=1}^{p^{n}} S^{a t}$, we get that the action of the generator of $C_{t}$ is $C_{p^{n}}$-equivariantly homotopic to the identity.

Putting this together, we get that the action of a generator of $C_{t}$ on all of $U^{t p^{n}}(A ; A \otimes$ $\left.S^{a}\right)$ is $C_{p^{n}}$-equivariantly homotopic to the identity. Thus the induced action on $U^{t p^{n}}(A ; A \otimes$ $\left.S^{a}\right)_{h C_{p^{n}}}$ is homotopic to the identity, and so is the induced action on $\left(U^{t p^{n}}(A ; A \otimes\right.$ $\left.\left.S^{a}\right)_{h C_{p^{n}}}\right)_{p}^{\wedge}$.

Lemma (6.2) Let $t$ be an even number coprime to the prime $p$. Then for a odd,

$$
\left(\left(U^{t p^{b}}\left(A ; A \otimes S^{a}\right)^{C_{p} b}\right)_{p}^{\wedge}\right)_{h C_{t}} \simeq *
$$

for any $b \geq 0$, and so

$$
\left(\left(\operatorname{holim}_{\infty \leftarrow n} U^{t p^{n}}(A ; M)^{C_{p^{n}}}\right)_{p}^{\wedge}\right)_{h C_{t}} \simeq *
$$

Proof. As in the proof of Lemma (6.1) above, $U^{t p^{b}}\left(A ; A \otimes S^{a}\right)^{C_{p^{b}}}$ is built by successive fibrations (which, for spectra, are cofibrations) from pieces of the form $U^{t p^{n}}\left(A ; A \otimes S^{a}\right)_{h C_{p^{n}}}$ for $n \leq b$. Thus $\left(\left(U^{t p^{b}}\left(A ; A \otimes S^{a}\right)^{C_{p^{b}}}\right)_{p}^{\wedge}\right)_{h C_{t}}$ is built by successive cofibrations from pieces of the form $\left(\left(U^{t p^{n}}\left(A ; A \otimes S^{a}\right)_{h C_{p^{n}}}\right)_{p}^{\wedge}\right)_{h C_{t}}$ for $n \leq b$.

Since $t$ is coprime to $p$, the spectral sequence

$$
\left.H_{*}\left(C_{t} ; \pi_{*}\left(\left(U^{t p^{n}}\left(A ; A \otimes S^{a}\right)_{h C_{p^{n}}}\right)_{p}^{\wedge}\right)\right) \Rightarrow \pi_{*}\left(\left(\left(U^{t p^{n}}\left(A ; A \otimes S^{a}\right)_{h C_{p^{n}}}\right)_{p}^{\wedge}\right)\right)_{h C_{t}}\right)
$$


is non-zero only in the zero'th column, which consists of $\pi_{*}\left(\left(U^{t p^{n}}\left(A ; A \otimes S^{a}\right)_{h C_{p^{n}}}\right)_{p}^{\wedge}\right)_{C_{t}}$. We will show that $C_{t}$ acts as multiplication by -1 on $\pi_{*}\left(\left(U^{t p^{n}}\left(A ; A \otimes S^{a}\right)_{h C_{p^{n}}}\right)_{p}^{\wedge}\right)$. Then taking the $C_{t}$ coinvariants is the same as quotienting out by the image of multiplication by $1-(-1)=2$, which is invertible after the $p$-completion. Therefore anything built from the $\left(\left(U^{t p^{n}}\left(A ; A \otimes S^{a}\right)_{h C_{p^{n}}}\right)_{p}^{\wedge}\right)_{h C_{t}}$ by cofibrations will have to be contractible.

As in the proof of Lemma (6.1), $U^{t p^{n}}\left(A ; A \otimes S^{a}\right) \simeq U^{t p^{n}}(A ; A) \wedge \bigwedge_{i=1}^{t p^{n}} S^{a} C_{t p^{n-}}$ equivariantly. As before, the $C_{t}$ action on $U^{t p^{n}}(A ; A)$ is $C_{p^{n}}$-equivariantly homotopic to the identity. Since $t$ is even and $a$ is odd, the generator of $C_{t}$ acts on each of the $p^{n}$ coordinates of $S^{a t p^{n}} \cong \bigwedge_{i=1}^{p^{n}} S^{a t}$ by a map of degree -1 : it cycles $t$ blocks of $a$ coordinates, and $(t-1) a$ is odd. Since $t$ and therefore also at are even, the antipodal map on each $S^{a t}$ is also of degree -1 . So the action of a generator of $C_{t}$ on $\bigwedge_{i=1}^{p^{n}} S^{a t}$ is $C_{p^{n}}$-equivariantly homotopic to the antipodal map.

We get that the action of a generator of $C_{t}$ on $U^{t p^{n}}\left(A ; A \otimes S^{a}\right) \simeq U^{t p^{n}}(A ; A) \wedge \bigwedge_{i=1}^{t p^{n}} S^{a}$

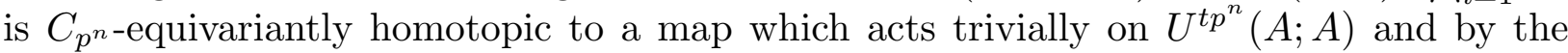
quotient $C_{2}$ action (via the antipodal map) on the sphere. Therefore the action of a generator of $C_{t}$ on $U^{t p^{n}}\left(A ; A \otimes S^{a}\right)_{h C_{p^{b}}}$ is homotopic to the action of a generator of $C_{2}$ induced by taking the identity on $U^{t p^{n}}(A ; A)$ and the antipodal map on the sphere.

The antipodal map induces multiplication by -1 on $\tilde{H}_{*}\left(S^{a t p^{n}} ; \mathbb{Z}\right)$, so it induces multiplication by -1 on $\pi_{*}\left(U^{t p^{n}}\left(A ; A \otimes S^{a}\right)\right) \cong \tilde{H}_{*}\left(S^{a t p^{n}} ; \pi_{*}\left(U^{t p^{n}}(A ; A)\right)\right)$. We have a spectral sequence

$$
H_{*}\left(C_{p^{n}} ; \pi_{*}\left(U^{t p^{n}}\left(A ; A \otimes S^{a}\right)\right)\right) \Rightarrow \pi_{*}\left(U^{t p^{n}}\left(A ; A \otimes S^{a}\right)_{h C_{p^{n}}}\right) .
$$

The action of the generator of $C_{2}$ induces multiplication by -1 on the $E^{2}$ term, and therefore also on the $E^{\infty}$ term, of this spectral sequence. We are however interested in its effect on $\pi_{*}\left(U^{t p^{n}}\left(A ; A \otimes S^{a}\right)_{h C_{p^{n}}}\right)$.

So what we have is a $C_{2}$ action on a filtered graded abelian group which induces multiplication by -1 on the associated graded complex, and we need to see what it does on the original group. Let $T$ denote the generator of $C_{2}$, and let $F_{n}$ denote the $n$ 'th filtration. Then on $F_{0}, C_{2}$ acts as multiplication by -1 . For any $x_{1} \in F_{1}$, we know that $T x_{1}=-x_{1}+x_{0}$ for some $x_{0} \in F_{0}$, and so $T^{2} x_{1}=T\left(-x_{1}\right)+T x_{0}=x_{1}-2 x_{0}$, but $T^{2} x_{1}=x_{1}$ so $2 x_{0}=0$. For any $x_{2} \in F_{2}, T x_{2}=-x_{2}+x_{1}$ for some $x_{1} \in F_{1}$ for which $T x_{1}=-x_{1}+x_{0}$ for some $x_{0} \in F_{0}$ satisfying $2 x_{0}=0$. We get $T^{2} x_{2}=2_{2}-2 x_{1}+x_{0}=x_{2}$ implying $2 x_{1}=x_{0}$ and so $4 x_{1}=0$. In general we get that for all $x_{n} \in F_{n}, T x_{n}=-x_{n}+x_{n-1}$ where $2^{n} x_{n-1}=0$.

Once we take the $p$ completion, since $p$ is odd all the 2-torsion will be eliminated. We get that the generator of $C_{2}$ (which acts homotopically to the generator of $C_{t}$ which we were originally looking at) acts as multiplication by -1 on $\pi_{*}\left(\left(U^{t p^{n}}\left(A ; A \otimes S^{a}\right)_{h C_{p^{n}}}\right)_{p}^{\wedge}\right)$.

\section{$\S 7$. The Reduced Algebraic K-Theory of Extensions of a Perfect Characteristic $p$ Field by Direct Sums of Itself}

In this section we will calculate the completion at $p$ of $\tilde{K}\left(A \ltimes\left(A^{\oplus k}\right)\right)$, the reduced algebraic K-theory of the trivial square-zero extension of $A$ by a direct sum of finitely 
many copies of itself, where $A$ is a perfect field of characteristic $p$. The case $k=1$, the dual numbers over $A$, has been done by Hesselholt and Madsen [6]. They calculated $\tilde{K}(A[\epsilon])_{p}^{\wedge}$ by looking at $\operatorname{TC}(A[\epsilon])$. Here we do a different calculation: we use $W$, and what we need to know about $A$ is that $W^{(p)}(A ; A)$ in our notation (which is $\mathrm{TR}^{(p)}$ in the notation of [1] and [6]) is known and its homotopy is concentrated in dimension 0. Theorem 5.5 in [6] gives this for $A$ a perfect field of characteristic $p$.

By [2], for any $A$-bimodule $M$

$$
K(A \ltimes M) \simeq K(A ; B . M) \simeq K\left(A ; M \otimes S^{1}\right) .
$$

The leftmost term maps to $K(A)$ via the augmentation $A \ltimes M \rightarrow A$; the right two terms map to $K(A ; 0) \cong K(A)$ via the map $M \rightarrow 0$. By the functoriality in $M$ of the correspondence (7.0.1), these reduction maps (both of which are split by the maps induced by $0 \rightarrow M)$ are compatible and give an equivalence of the reduced theories

$$
\tilde{K}(A \ltimes M) \simeq \tilde{K}\left(A ; M \otimes S^{1}\right) .
$$

But by [8], for any $A$-bimodule $M$,

$$
\tilde{K}\left(A ; M \otimes S^{1}\right) \simeq W\left(A ; M \otimes S^{1}\right) .
$$

Using the decomposition from Remark (3.6), we can decompose $W\left(A ; M \otimes S^{1}\right)$ into a product and get that

$$
\tilde{K}(A \ltimes M)_{p}^{\wedge} \simeq \prod_{(t, p)=1}\left(\left(\operatorname{holim}_{\infty \leftarrow n} U^{t p^{n}}\left(A ; M \otimes S^{1}\right)^{C_{p^{n}}}\right)_{p}^{\wedge}\right)_{h C_{t}} .
$$

When the homotopy of $\operatorname{holim}_{\infty \leftarrow n} U^{r p^{n}}(A ; M)^{C_{p^{n}}} \cong W\left(A ; M^{\hat{\otimes}_{A} r}\right)$ is concentrated in dimension 0 for all $r \geq 0$, we can use Theorem (5.3) to give us an explicit formula for the homotopy groups of holim $\infty_{\infty \leftarrow n} U^{t p^{n}}\left(A ; M \otimes S^{1}\right)^{C_{p^{n}}}$, from which we would also know the homotopy groups of its $p$ completion, but we would still need to calculate the effect of taking the homotopy quotients.

For $M=A^{\oplus k}$, however, we can use the decomposition formula from Theorem (2.2) to get our answer in a closed form: We have

$$
\tilde{K}\left(A \ltimes\left(A^{\oplus k}\right)\right) \simeq \tilde{K}\left(A ;\left(A^{\oplus k}\right) \otimes S^{1}\right) \simeq W\left(A ;\left(A^{\oplus k}\right) \otimes S^{1}\right) \simeq W\left(A ; \bigoplus_{i=1}^{k} A \otimes S^{1}\right) .
$$

As explained in the proof of Application (2.3),

$$
W\left(A ; \bigoplus_{i=1}^{k} A \otimes S^{1}\right) \simeq W\left(A ; \bigvee_{i=1}^{k} A \otimes S^{1}\right)
$$


and by Theorem (2.2),

$$
W\left(A ; \bigvee_{i=1}^{k} A \otimes S^{1}\right) \simeq \prod_{a=1}^{\infty} \prod_{\left.[f] \in \tilde{w}_{a}(\underline{k})\right) / C_{a}} W\left(A ;\left(A \otimes S^{1}\right)^{\hat{\otimes}_{A} a}\right)
$$

Since $\left(A \otimes S^{1}\right)^{\hat{\otimes}_{A} a} \simeq A^{\hat{\otimes}_{A} a} \otimes\left(S^{1}\right)^{\wedge a} \simeq A \otimes S^{a}$, putting all the above equivalences together, we get that

$$
\tilde{K}\left(A \ltimes\left(A^{\oplus k}\right)\right) \simeq \prod_{a=1}^{\infty} \prod_{\left.[f] \in \tilde{w}_{a}(\underline{k})\right) / C_{a}} W\left(A ; A \otimes S^{a}\right) .
$$

Now, if we complete at $p$, we can use Remark (3.6) on each $W\left(A ; A \otimes S^{a}\right)$ to decompose

$$
W\left(A ; A \otimes S^{a}\right)_{p}^{\wedge} \simeq \prod_{(t, p)=1}\left(\left(\operatorname{holim}_{\infty \leftarrow n} U^{t p^{n}}\left(A ; A \otimes S^{a}\right)^{C_{p^{n}}}\right)_{p}^{\wedge}\right)_{h C_{t}}
$$

and Lemmas (6.1) and (6.2) analyze the factors. We get

Corollary (7.1) For any FSP A, (i) If $p$ is an odd prime and $a$ is odd,

$$
W\left(A ; A \otimes S^{a}\right)_{p}^{\wedge} \simeq \prod_{(t, 2 p)=1} W^{(p)}\left(A ; A \otimes S^{a t}\right)_{p}^{\wedge}
$$

(ii) If $p$ is an odd prime and $a$ is even,

$$
W\left(A ; A \otimes S^{a}\right)_{p}^{\wedge} \simeq \prod_{(t, p)=1} W^{(p)}\left(A ; A \otimes S^{a t}\right)_{p}^{\wedge}
$$

(iii) If $p=2$,

$$
W\left(A ; A \otimes S^{a}\right)_{2}^{\wedge} \simeq \prod_{(t, 2)=1} W^{(2)}\left(A ; A \otimes S^{a t}\right)_{2}^{\wedge}
$$

To encode the resulting decomposition of $\tilde{K}\left(A \ltimes\left(A^{\oplus k}\right)\right)_{p}^{\wedge}$, we will use the Möbius $\mu$-function defined on positive integers $n$ by

$$
\mu(n)= \begin{cases}0 & \text { if } n \text { has a nontrivial square factor } \\ (-1)^{k} & \text { if } n=p_{1} p_{2} \cdots p_{k} \text { for distinct primes } p_{i} .\end{cases}
$$

Recall that $\sum_{d \mid a} \mu\left(\frac{a}{d}\right) k^{d}$ counts the non-periodic words of length $a$ in $k$ symbols. 
Theorem (7.2) For any FSP A and prime p,

$$
K\left(A \ltimes\left(A^{\oplus k}\right)\right)_{p}^{\wedge} \simeq W\left(A ;\left(A^{\oplus k}\right) \otimes S^{1}\right)_{p}^{\wedge} \simeq \prod_{i=1}^{\infty}\left(W^{(p)}\left(A ; A \otimes S^{i}\right)_{p}^{\wedge}\right)^{m_{i}}
$$

where for $i$ odd or if $p=2$,

$$
m_{i}=\sum_{\substack{t \mid i \\(t, p)=1}} \frac{t}{i} \sum_{\left.d\right|_{\frac{i}{t}}} \mu\left(\frac{i}{t d}\right) k^{d}
$$

while for $i$ even and $p$ odd,

$$
m_{i}=\sum_{\substack{t \mid \frac{i}{2} \\(t, p)=1}} \frac{t}{i} \sum_{d \mid \frac{i}{t}} \mu\left(\frac{i}{t d}\right) k^{d}
$$

Proof. By Corollary (7.1) above, for $p$ odd

$$
\begin{gathered}
W\left(A ; A^{\oplus k} \otimes S^{1}\right)_{p}^{\wedge} \simeq \prod_{a=1}^{\infty} \prod_{[f] \in \tilde{w}_{a}(\underline{\underline{k}}) / C_{a}} W\left(A ; A \otimes S^{a}\right)_{p}^{\wedge} \\
\simeq \prod_{b=1}^{\infty} \prod_{[f] \in \tilde{w}_{2 b+1}(\underline{\underline{k}}) / C_{2 b+1}} \prod_{(t, 2 p)=1} W^{(p)}\left(A ; A \otimes S^{(2 b+1) t}\right)_{p}^{\wedge} \\
\quad \times \prod_{b=1}^{\infty} \prod_{[f] \in \tilde{w}_{2 b}(\underline{k}) / C_{2 b}} \prod_{(t, p)=1} W^{(p)}\left(A ; A \otimes S^{2 b t}\right)_{p}^{\wedge} \\
\simeq \prod_{i=1}^{\infty}\left(W^{(p)}\left(A ; A \otimes S^{i}\right)_{p}^{\wedge}\right)^{m_{i}}
\end{gathered}
$$

where for odd $i$, since a copy of $W^{(p)}\left(A ; A \otimes S^{i}\right)$ could come only from the first part of the product corresponding to $a=2 b+1$ odd,

$$
m_{i}=\sum_{\substack{t \mid i \\(t, p)=1}} \frac{\left|\tilde{w}_{\frac{i}{t}}(\underline{k})\right|}{\frac{i}{t}}=\sum_{\substack{t \mid i \\(t, p)=1}} \frac{t}{i} \sum_{d \mid \frac{i}{t}} \mu\left(\frac{i}{t d}\right) k^{d}
$$

for $\mu$ as in (7.1.1), while for $i$ even, since a copy of $W^{(p)}\left(A ; A \otimes S^{i}\right)$ could come only from the second part of the product corresponding to $a=2 b$ even,

$$
m_{i}=\sum_{\substack{t \mid \frac{i}{2} \\(t, p)=1}} \frac{\left|\tilde{w}_{\frac{i}{t}}(\underline{k})\right|}{\frac{i}{t}}=\sum_{\substack{t \mid \frac{i}{2} \\(t, p)=1}} \frac{t}{i} \sum_{d \mid \frac{i}{t}} \mu\left(\frac{i}{t d}\right) k^{d} .
$$


For $p=2$, by Corollary (7.1) again,

$$
\begin{aligned}
W\left(A ; A^{\oplus k} \otimes S^{1}\right)_{2}^{\wedge} \simeq \prod_{a=1}^{\infty} \prod_{[f] \in \tilde{w}_{a}(\underline{k}) / C_{a}} W\left(A ; A \otimes S^{a}\right)_{2}^{\wedge} \\
\simeq \prod_{a=1}^{\infty} \prod_{[f] \in \tilde{w}_{a}(\underline{k}) / C_{a}} \prod_{(t, 2)=1} W^{(2)}\left(A ; A \otimes S^{a t}\right)_{2}^{\wedge} \cong \prod_{i=1}^{\infty}\left(W^{(2)}\left(A ; A \otimes S^{i}\right)_{2}^{\wedge}\right)^{m_{i}}
\end{aligned}
$$

where

$$
m_{i}=\sum_{\substack{t \mid i \\(t, 2)=1}} \frac{\left|\tilde{w}_{\frac{i}{t}}(\underline{k})\right|}{\frac{i}{t}}=\sum_{\substack{t \mid i \\(t, 2)=1}} \frac{t}{i} \sum_{d \mid \frac{i}{t}} \mu\left(\frac{i}{t d}\right) k^{d}
$$

When $A$ is an FSP for which the homotopy of $W^{(p)}(A ; A)$ is concentrated in dimension zero we then can use Theorem (5.3) to explicitly calculate the homotopy groups of $W^{(p)}\left(A ; A \otimes S^{i}\right)$ for all $i$. This completes the calculation of $\pi_{*}\left(\tilde{K}\left(A \ltimes\left(A^{\oplus k}\right)\right)_{p}^{\wedge}\right)$ for such FSPs $A$.

The actual formulae for $\pi_{*}\left(\tilde{K}\left(A \ltimes\left(A^{\oplus k}\right)\right)_{p}^{\wedge}\right)$ are in general a little cumbersome to write out, but in the special case where $A$ is a perfect field of characteristic $p>0$, we use the results of Corollaries (5.5) and (5.6), and get

Theorem (7.3) Let $A$ be a perfect field of odd characteristic p; we will use the notation $A$ also to denote its associated FSP. Let $W(A)=\pi_{0}\left(W^{(p)}(A ; A)\right)$ denote the ring of p-typical Witt vectors of $A$. Then for $j$ odd,

$$
\pi_{j}\left(K\left(A \ltimes\left(A^{\oplus k}\right)\right)_{p}^{\wedge}\right) \cong \bigoplus_{\substack{2 i+1 \leq j \\(2 i+1) p^{r-1}<j \leq(2 i+1) p^{r}}}\left(W(A) / p^{r} W(A)\right)^{m_{2 i+1}},
$$

and for $j$ even,

$$
\pi_{j}\left(K\left(A \ltimes\left(A^{\oplus k}\right)\right)_{p}^{\wedge}\right) \cong \bigoplus_{\substack{2 i \leq j \\ 2 i p^{r-1}<j \leq 2 i p^{r}}}\left(W(A) / p^{r} W(A)\right)^{m_{2 i}}
$$

where

$$
m_{2 i+1}=\sum_{\substack{t \mid 2 i+1 \\(t, p)=1}} \frac{t}{2 i+1} \sum_{d \mid \frac{2 i+1}{t}} \mu\left(\frac{2 i+1}{t d}\right) k^{d}
$$

and

$$
m_{2 i}=\sum_{\substack{t \mid i \\(t, p)=1}} \frac{t}{2 i} \sum_{d \mid \frac{2 i}{t}} \mu\left(\frac{2 i}{t d}\right) k^{d}
$$


Theorem (7.4) Let $A$ be a perfect field of characteristic 2 ; we will use the notation $A$ also to denote its associated FSP. Let $W(A)=\pi_{0}\left(W^{(2)}(A ; A)\right)$ denote the ring of 2-typical Witt vectors of $A$. Then for $j$ odd,

$$
\pi_{j}\left(K\left(A \ltimes\left(A^{\oplus k}\right)\right)_{2}^{\wedge}\right) \cong \bigoplus_{2 i+1 \leq j}(W / 2 W)^{m_{2 i+1}},
$$

and for $j$ even,

$$
\pi_{j}\left(K\left(A \ltimes\left(A^{\oplus k}\right)\right)_{2}^{\wedge}\right) \cong \bigoplus_{\substack{2 i \leq j \\ i 2^{r}<j \leq i 2^{r+1}}}\left(W / 2^{r} W\right)^{m_{2 i}}
$$

where

$$
m_{i}=\sum_{\substack{t \mid i \\(t, p)=1}} \frac{t}{i} \sum_{d \mid \frac{i}{t}} \mu\left(\frac{i}{t d}\right) k^{d} .
$$

Example (7.5) As a sample of other calculations which can be done with our methods, we re-calculate $\tilde{K}(\mathbb{Z} \ltimes \mathbb{Q})$, which is known by work of T. Goodwillie in [7] to be equivalent to the relative cyclic homology of $\mathbb{Z} \ltimes \mathbb{Q}$ over $\mathbb{Z}$ (shifted up by one degree) which can be calculated directly, and is also given by his formula (IV.1.6). By Theorem (3.2), $W\left(\mathbb{Z} ; \mathbb{Q} \otimes S^{1}\right)$ actually splits as the product of its layers, so we group the layers according to their prime-to-2 part to obtain

$$
\tilde{K}(\mathbb{Z} \ltimes \mathbb{Q}) \simeq W\left(\mathbb{Z} ; \mathbb{Q} \otimes S^{1}\right) \simeq \prod_{(t, 2)=1} W^{(2)}\left(\mathbb{Z} ; \mathbb{Q} \otimes S^{t}\right)_{h C_{t}} .
$$

Choosing the prime 2 saves a few lines in the argument, but this calculation can be done with any prime.

Observe that since $H \mathbb{Z} \wedge H \mathbb{Q} \rightarrow H \mathbb{Q} \wedge H \mathbb{Q}$ induces a homology equivalence, hence a weak homotopy equivalence, $U^{n}\left(\mathbb{Z} ; \mathbb{Q} \otimes S^{t}\right) \simeq U^{n}\left(\mathbb{Q} ; \mathbb{Q} \otimes S^{t}\right) C_{n}$-equivariantly for all $n$.

By the method of Lemma (6.1)(which uses only the fact that multiplication by $t$ acts invertibly on the homotopy groups $), W^{(2)}\left(\mathbb{Q} ; \mathbb{Q} \otimes S^{t}\right) \stackrel{\sim}{\longrightarrow} W^{(2)}\left(\mathbb{Q} ; \mathbb{Q} \otimes S^{t}\right)_{h C_{t}}$ for all $t$ odd and so

$$
\tilde{K}(\mathbb{Z} \ltimes \mathbb{Q}) \simeq \prod_{(t, 2)=1} W^{(2)}\left(\mathbb{Q} ; \mathbb{Q} \otimes S^{t}\right) .
$$

Now by Corollary (5.4),

$$
\pi_{*}\left(W^{(2)}\left(\mathbb{Q} ; \mathbb{Q} \otimes S^{t}\right)\right) \cong \begin{cases}\operatorname{coker}\left(v_{2}\right) & \text { if } *=t \\ \operatorname{ker}\left(v_{2}\right) & \text { if } *=t+1 \\ 0 & \text { otherwise }\end{cases}
$$

since multiplication by 2 is an isomorphism over the rationals. In terms of the Witt coordinates, on the 2-typical Witt vectors of $A, v_{2}\left(a_{0}, a_{1}, a_{2}, \ldots\right)=\left(0, a_{0}, a_{1}, a_{2}, \ldots\right)$. This shows us that $\pi_{*}\left(W^{(2)}\left(\mathbb{Q} ; \mathbb{Q} \otimes S^{t}\right)\right)$ is $\mathbb{Q}$ in dimension $t$ and vanishes elsewhere, so $W^{(2)}\left(\mathbb{Q} ; \mathbb{Q} \otimes S^{t}\right)$ is the Eilenberg-Mac Lane spectrum $K(\mathbb{Q}, t)$ and

$$
\tilde{K}(\mathbb{Z} \times \mathbb{Q}) \simeq \prod_{(t, 2)=1} K(\mathbb{Q}, t) .
$$




\section{REFERENCES}

[1] M. Bökstedt, W.-C. Hsiang, I. Madsen, The Cyclotomic trace and algebraic K-theory of spaces, Invent. Math. 111 (1993) 465-540.

[2] B. I. Dundas, R. McCarthy, Stable K-theory and topological Hochschild homology, Ann. of Math. 140, (1994) 685-701.

[3] B. Dundas, R. McCarthy, Topological Hochschild homology of ring functors and exact categories, J. Pure Appl. Algebra 109 (1996) no. 3, 231-294.

[4] J. P. C. Greenlees, J. P. May, Generalized Tate Cohomology, Mem. Amer. Math. Soc. 113 (1995) no. 543, viii+178 pp.

[5] J. P. C. Greenlees, J. P. May, Equivariant stable homotopy theory, Handbook of Algebraic Topology, ed. I. M. James, 279-325, North Holland, Amsterdam 1995.

[6] L. Hesselholt, I. Madsen, On the K-theory of finite algebras over Witt vectors of perfect fields, Topology 36 (1997) no. 1, 29-101.

[7] T. Goodwillie, Relative Algebraic K-theory, Ann. of Math. 124 (2) (1986) no. 2, $347-402$.

[8] A. Lindenstrauss, R. McCarthy, On the Taylor Tower of Relative K-theory, preprint. 